\title{
Synthesis and Closed-loop Recycling of Self-immolative Poly(Dithiothreitol)
}

\author{
Sunirmal Pal,,${ }^{\mathrm{a},}$ Andreas Sommerfeldt, ${ }^{\mathrm{a} \ddagger}$ Maiken Berglund Davidsen, ${ }^{\mathrm{a}}$ Mogens Hinge, ${ }^{\mathrm{b}}$ Steen Uttrup Pedersen, ${ }^{\mathrm{a}}$ \\ Kim Daasbjerg ${ }^{\mathrm{a}, *}$
}

[a] Department of Chemistry and Interdisciplinary Nanoscience Center (iNANO), Aarhus University, Langelandsgade 140,8000 Aarhus C, Denmark

[b] Department of Engineering, Plastic and Polymer Engineering, Aabogade 40a, 8200 Aarhus N, Denmark

[†] These authors contributed equally.

Corresponding author: kdaa@chem.au.dk

\begin{abstract}
Self-immolative polymers (SIPs) are promising members of the emerging class of recyclable polymers with the ability to end-to-end depolymerize to their monomers. Unfortunately, SIPs are often synthesized by cumbersome procedures at low temperatures in protected atmosphere. In this study, a SIP with a novel poly(disulfide) backbone is introduced, using DL-dithiothreitol (DTT) as monomer. Remarkably, poly(DTT) can be produced by solid-state polymerization in a robust and easily scalable process by mechanically mixing DTT with 2,2'-dithiodipyridine as end-capping agent. The new polymer possesses good thermal and chemical stability but once its depolymerization is triggered, this proceeds smoothly within minutes to afford cyclic DTT due to a favorable intramolecular back-biting thiol-disulfide exchange reaction in the polymer backbone. As a proof-of-concept, the cyclic DTT waste was recovered, reduced to DTT monomer, and repolymerized in a closed-loop approach.
\end{abstract}


Supporting Information - Synthesis and Closed-Loop Recycling of Self-Immolative Poly(Dithiothreitol)

\section{Contents}

Materials ......

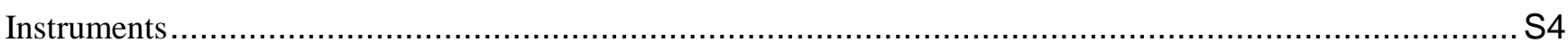

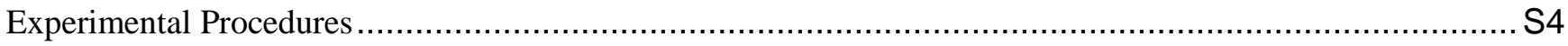

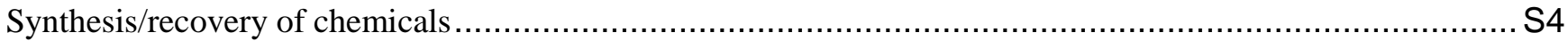

Recovery of 2,2'-dithiodipyridine (DTDP) from isolated pyridine-2-thiol ......................................... S4

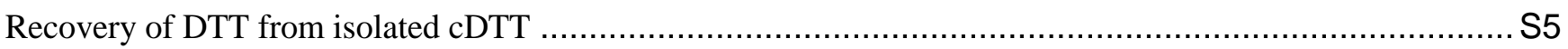

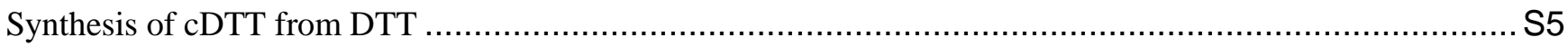

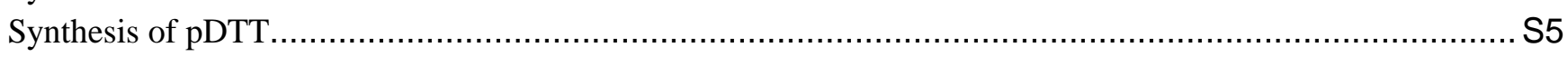

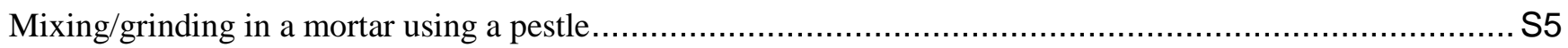

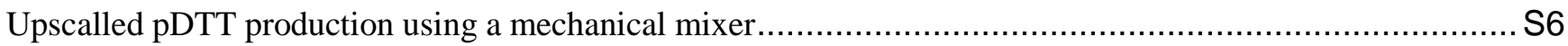

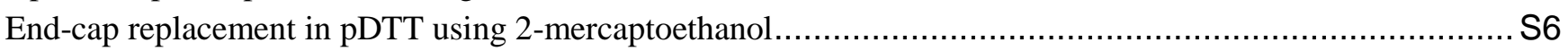

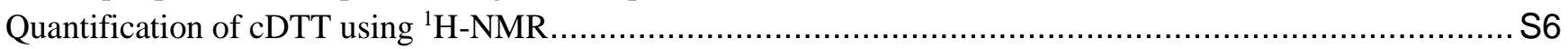

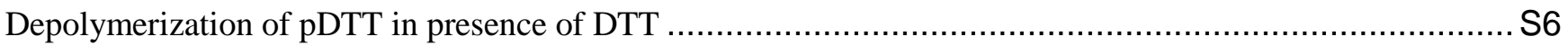

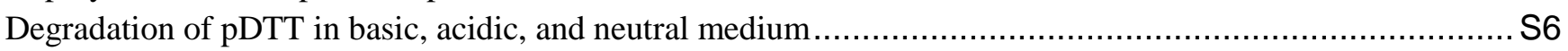

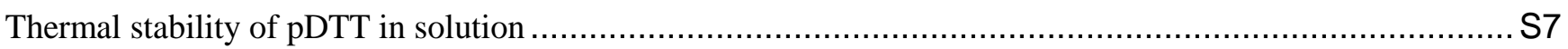

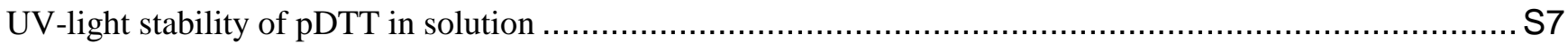

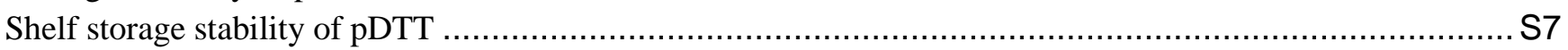

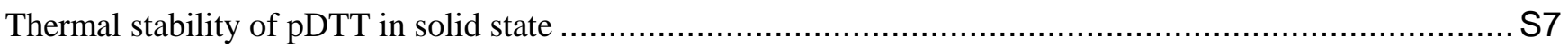

(I) ${ }^{1} \mathrm{H}-\mathrm{NMR}$ recording of the solid-state depolymerization of pDTT (undissolved in $\mathrm{D}_{2} \mathrm{O}$ ) in the presence of

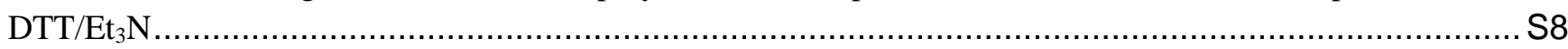

(II) Video recording of the solid-state depolymerization of pDTT (undissolved in $\mathrm{D}_{2} \mathrm{O}$ ) in the presence of

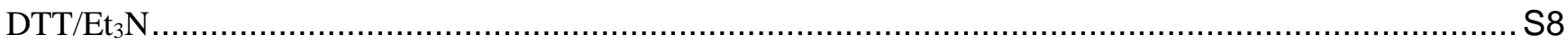

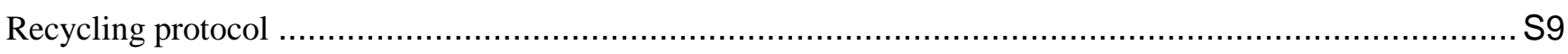

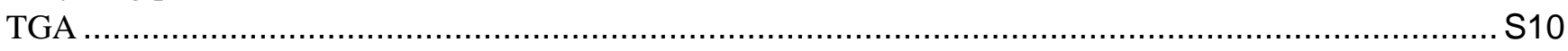

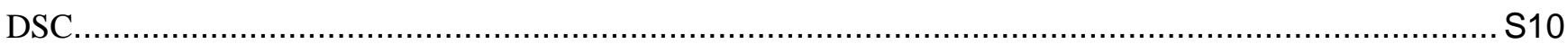

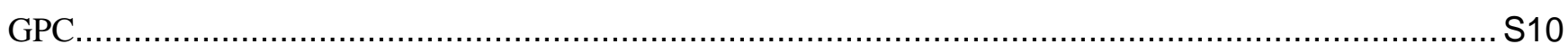

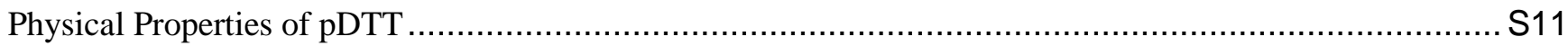

Thermal, photochemical, and chemical stability of pDTT in solution ................................................. S11

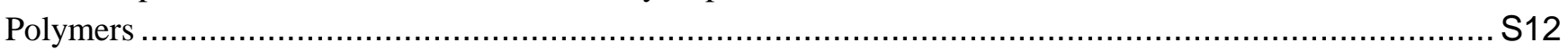

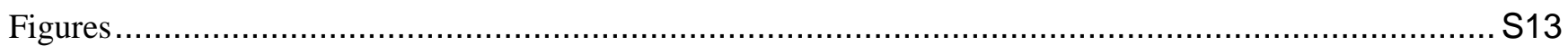

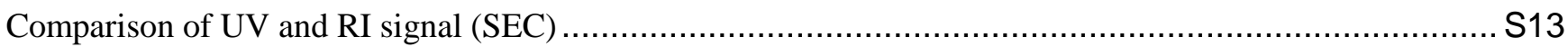

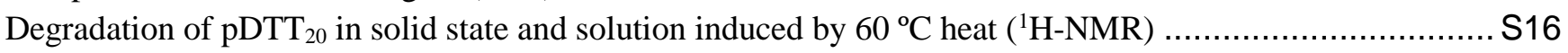

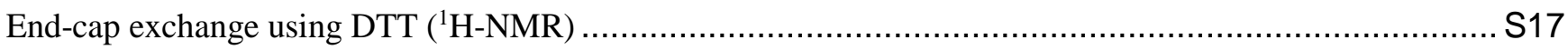

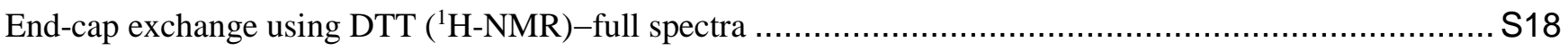

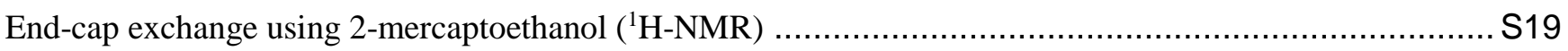

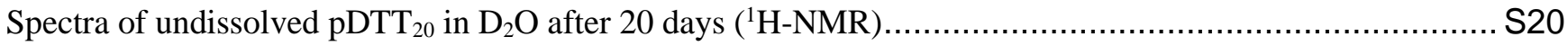

Solid-state depolymerization of $\mathrm{pDTT}_{20}$ submerged in $\mathrm{D}_{2} \mathrm{O}$ under inert argon atmosphere $\left({ }^{1} \mathrm{H}-\mathrm{NMR}\right) \ldots \ldots \ldots . \mathrm{S} 21$

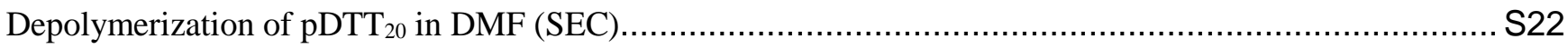

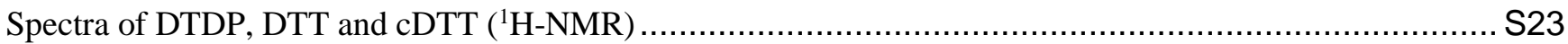

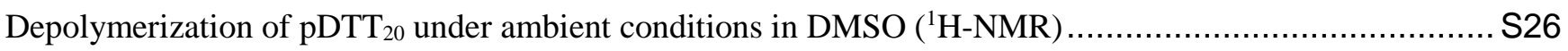

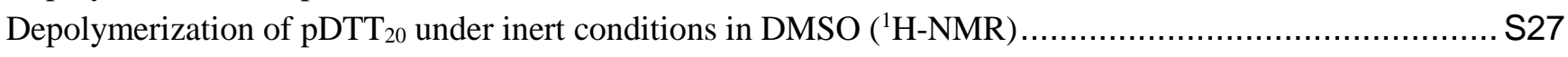


Supporting Information - Synthesis and Closed-Loop Recycling of Self-Immolative Poly(Dithiothreitol)

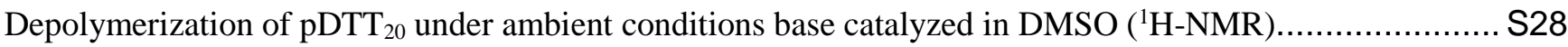

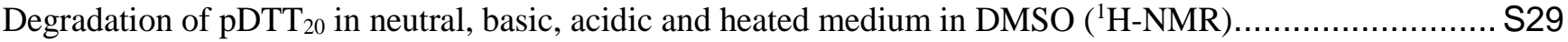

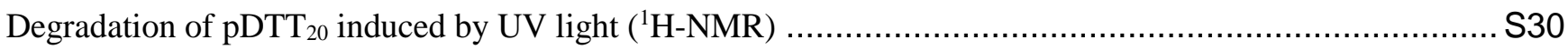

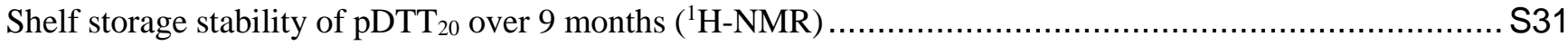

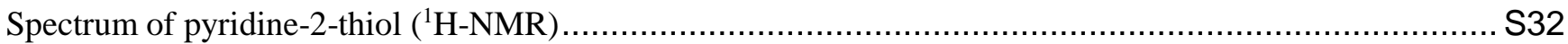

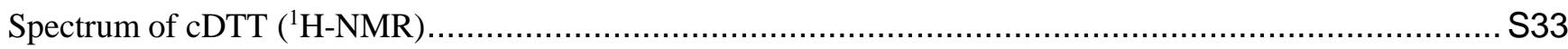

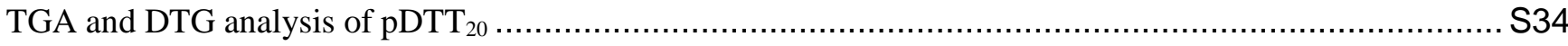

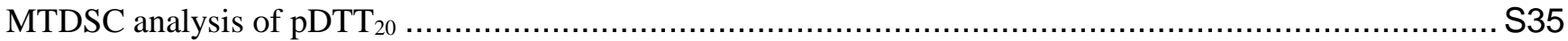

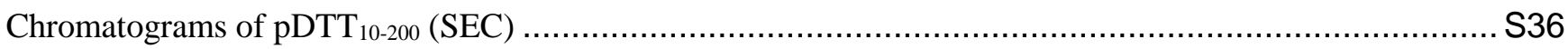

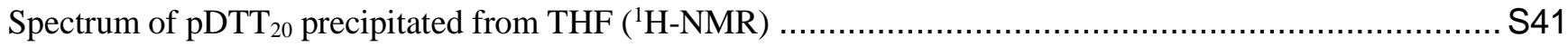

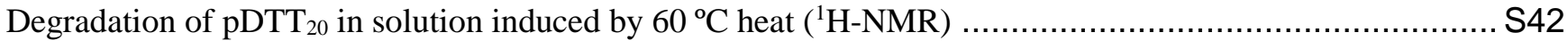

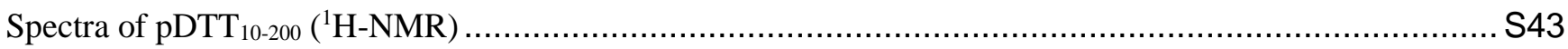

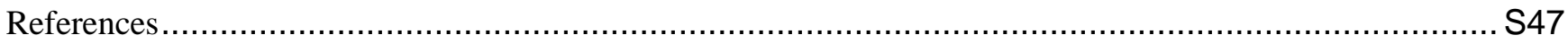

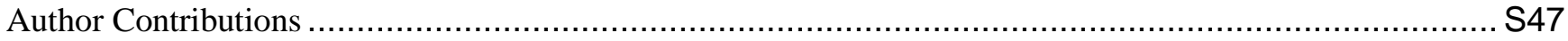


Supporting Information - Synthesis and Closed-Loop Recycling of Self-Immolative Poly(Dithiothreitol)

\section{Materials}

All synthetic procedures were carried out under ambient conditions unless otherwise stated. Most solvents and chemicals were purchased from Sigma Aldrich and used without further purification. 2,2'-Dithiodipyridine (DTDP) was either purchased from Sigma Aldrich, synthesized from pyridine-2-thiol, or recovered from earlier polymerizations (vide infra). Dithiothreitol (DTT) was purchased from Fischer Scientific and used without further purification or synthesized from cyclic DTT (cDTT) recovered from the depolymerization.

\section{Instruments}

${ }^{1} \mathrm{H}-\mathrm{NMR}$ spectra were recorded using a Bruker $400 \mathrm{MHz}$ spectrometer with $\mathrm{CDCl}_{3}, \mathrm{DMSO}-d_{6}$ or $\mathrm{D}_{2} \mathrm{O}$ as internal reference. Size exclusion chromatography was performed using a system comprising a LC-20AD Shimadzu HPLC pump, a Shimadzu RID-10A refractive index detector, and a DAWN HELEOS 8 light scattering detector from Wyatt. The detector was a SPD-M20A PDA, equipped with a Mz-Gel SDplus Linear column $(8 \times 300 \mathrm{~mm})$ using $5 \mu \mathrm{m}$ particles from MZ-Analysentechnik to provide an effective molecular weight range of 1'000-1'000'000 Da. $\mathrm{N}, \mathrm{N}$-Dimethylformamide (DMF) containing $10 \mathrm{mM} \mathrm{LiBr}$ was employed as solvent. For molar weight calculations, the average $\mathrm{dn} / \mathrm{dc}$ value was calculated to be 0.1146 determined by assuming full mass recovery on samples without solvents as justified from the recorded ${ }^{1} \mathrm{H}-\mathrm{NMR}$ spectra. UV degradation was performed using a UV-LED lamp Spectroline ${ }^{\circledR}$ TRITAN-365F/XF-F1 mounted at a fixed distance of $10 \mathrm{~cm}$ from an NMR tube sample holder. Thermogravimetric analysis (TGA) was carried out with a NETZSCH TG 209 F1 Libra TGA (NETZSCH, Germany). Differential scanning calorimetry (DSC) analysis was performed with a PerkinElmer DSC 8000. The mechanical mixer used for the upscaling experiments was a DAC 150.1 FVZ-K SpeedMixer from Hauchchild.

\section{Experimental Procedures}

\section{Synthesis/recovery of chemicals}

Recovery of 2,2'-dithiodipyridine (DTDP) from isolated pyridine-2-thiol<smiles>CCOC(=O)CC(C)(O)C(=O)OCC</smiles>

Pyridine-2-thiol $(25.0 \mathrm{~g}, 0.225 \mathrm{~mol})$ was added to a $250 \mathrm{ml}$ round bottom flask equipped with a magnetic stir bar and partly dissolved in EtOAc $(125 \mathrm{ml})$ and cooled to $0{ }^{\circ} \mathrm{C} . \mathrm{H}_{2} \mathrm{O}_{2}(33 \% \mathrm{w} / \mathrm{w} ; 10.9 \mathrm{ml}, 0.1115 \mathrm{~mol})$ was added dropwise over $60 \mathrm{~min}$. The substrate became fully dissolved during the addition of $\mathrm{H}_{2} \mathrm{O}_{2}$. The ice bath was removed and the solution was allowed to react at room temperature for $2 \mathrm{~h}$. The yellow colour of the solution fainted. Subsequently, $10 \mathrm{ml}$ water was added before the solution was transferred to a separatory funnel. The water phase was extracted twice with $50 \mathrm{ml}$ EtOAc and the combined organic phases reduced in vacuo. The resulting product was purified by coloumn chromatography using as eluent EtOAc/pentane $(3: 7 \mathrm{v} / \mathrm{v})$, yielding 2,2'-dithiodipyridine as a white powder $(22.9 \mathrm{~g}, 92 \%) .{ }^{1} \mathrm{H}-\mathrm{NMR} \mathrm{CDCl}_{3}(400 \mathrm{MHz}) \delta=8.47(\mathrm{dt}, 1 \mathrm{H}, J=4.7 \mathrm{~Hz}, 1.24 \mathrm{~Hz}), 7.62(\mathrm{~m}, 2 \mathrm{H})$, 7.11 (ddd, 1H, $J=6.7 \mathrm{~Hz}, 4.9 \mathrm{~Hz}, 2.1 \mathrm{~Hz}$ ) (Figure S11). 
Recovery of DTT from isolated cDTT<smiles>O[C@H](CS)[C@H](O)CS</smiles>

cDTT (0.100 g, $0.657 \mathrm{mmol})$ and tris-(2-carboxyethyl)phosphine (TCEP) (0.207 g, $0.827 \mathrm{mmol}$ ) were added to a $25 \mathrm{ml}$ round bottom flask equipped with a magnetic stir bar. The solids were dissolved in $10 \mathrm{ml} 0.1 \mathrm{M}$ phosphate buffered water ( $\mathrm{pH} 7)$. The reaction was stirred at room temperature for $2 \mathrm{~h}$ before addition of $50 \mu \mathrm{L} \mathrm{AcOH}$. The solvent was evaporated off, and the residue extracted three times with acetonitrile. The combined organic phases were filtered and reduced in vacuo yielding DTT $(95.0 \mathrm{mg}, 95 \%)$. ${ }^{1} \mathrm{H}-\mathrm{NMR}$ DMSO- $d_{6}(400 \mathrm{MHz}) \delta=4.82(\mathrm{~d}$, $2 \mathrm{H}, J=6.2 \mathrm{~Hz}), 3.52(\mathrm{~m}, 2 \mathrm{H}), 2.63-2.52(\mathrm{~m}, 2 \mathrm{H}), 2.18(\mathrm{dd}, 2 \mathrm{H}, J=9.5 \mathrm{~Hz}, 6.9 \mathrm{~Hz})$ (Figure S12).

Synthesis of cDTT from DTT<smiles>O[C@H](CS)[C@@H](O)CS</smiles><smiles>CCOC(=O)CC(N)O</smiles>

DTT ( $3.00 \mathrm{~g}, 19.4 \mathrm{mmol}$ ) was added to a $100 \mathrm{ml}$ round bottom flask equiped with a magnetic stir bar and dissolved in $25 \mathrm{ml} \mathrm{EtOAc}$ and cooled to $0{ }^{\circ} \mathrm{C}$. NaI $(29.0 \mathrm{mg}, 0.193 \mathrm{mmol})$ was added to the reaction mixture serving as a catalyst. $\mathrm{H}_{2} \mathrm{O}_{2}(33 \% \mathrm{w} / \mathrm{w} ; 2.0 \mathrm{ml}, 19.4 \mathrm{mmol})$ was added dropwise over $30 \mathrm{~min}$. Subsequently, the ice bath was removed after 30 min to allow the reaction to proceed at room temperature for $2 \mathrm{~h}$. The colour of the solution changed from colourless to brown. The reaction was quenched by addition of saturated $\mathrm{Na}_{2} \mathrm{~S}_{2} \mathrm{O}_{3}$ which made the solution colourless. It was diluted with addition of $20 \mathrm{ml}$ EtOAc, and the aqueous layer was removed using a separatory funnel. The organic layer was washed with $10 \mathrm{ml}$ brine, dried over $\mathrm{MgSO}_{4}$, and concentrated in vacuo to yield cDTT as a white powder $(2.70 \mathrm{~g}, 91 \%) .{ }^{1} \mathrm{H}-\mathrm{NMR}\left(400 \mathrm{MHz}, \mathrm{DMSO}-d_{6}\right) \delta=5.22(\mathrm{~d}, 2 \mathrm{H}, J=3.8 \mathrm{~Hz})$, $3.34(\mathrm{t}, 2 \mathrm{H}, J=4.5 \mathrm{~Hz}), 3.04(\mathrm{~d}, 2 \mathrm{H}, J=13.2 \mathrm{~Hz}), 2.84-2.60(\mathrm{~m}, 2 \mathrm{H})$ (Figure S13).

\section{Synthesis of pDTT}

Mixing/grinding in a mortar using a pestle

DTT ( 1 equiv.) and DTDP [ $(1+\xi)$ equiv.] were placed in a mortar. Typically, $0.1-1 \mathrm{~g}$ DTT was used and $\xi$ ranged from $0.025-0.1$ to hit $M_{\mathrm{n}, \text { theo }}=1740-30620 \mathrm{~g} \mathrm{~mol}^{-1}$ of the final polymer. The two solids were grinded to a paste using a pestle for $10 \mathrm{~min}$, or until the content turned from white solids to a yellow liquid and, further, into a pale yellow solid again. The paste was left in the mortar for additional 20-30 min (see provided video material). The polymer was dissolved in DMF and precipitated using $\mathrm{CHCl}_{3} /$ hexane $(1: 1 \mathrm{v} / \mathrm{v})$ repeatedly five times to remove the smaller molecular fragments. In between these precipitation steps, the polymer was separated by centrifugation and the solvent was decanted. Finally, the polymer was dried under vacuum ( 2 mbar) for at least $10 \mathrm{~h}$. To avoid DMF residues in the polymer, tetrahydrofuran (THF) could replace DMF in the purification procedure, although the disadvantage of THF was its inability to dissolve the pDTTs of highest DP. Yields were calculated from the weight of the isolated pDTT, relative to the amount of DTT and stoichiometric excess DTDP used. Note that the weight of pDTT was corrected from the contributions from DMF and chloroform as determined from the ${ }^{1} \mathrm{H}-\mathrm{NMR}$ estimated mass fractions. 
Supporting Information - Synthesis and Closed-Loop Recycling of Self-Immolative Poly(Dithiothreitol)

\section{Upscalled pDTT production using a mechanical mixer}

DTT $(5.00 \mathrm{~g}, 32.4 \mathrm{mmol})$ and DTDP $(7.50 \mathrm{~g}, 34.0 \mathrm{mmol})(\xi=0.05)$ were added to a $50 \mathrm{~mL}$ polypropylene cup, sealed with a lid and inserted into a Hauchchild SpeedMixer. Dry mixing of the compounds at 3000 rounds min ${ }^{-1}$ with vertical vibrations was carried out for $2 \mathrm{~min}$. This led to a solid-state reaction, producing pDTT and pyridine2-thiol in a hardened yellow paste. The paste was dissolved in $25 \mathrm{~mL}$ DMF in the propylene cup. The product was precipitated by dropwise addition into a mixture of $\mathrm{CHCl}_{3} /$ hexane $(1: 1 \mathrm{v} / \mathrm{v})$ in six different centrifugal tubes. The polymer was isolated through centrifugation, and repeatedly dissolved in DMF and precipitated into $\mathrm{CHCl}_{3} / \mathrm{hexane}$ $(1: 1 \mathrm{v} / \mathrm{v})$ twice, before removing residual solvent in vacuo $(<2 \mathrm{mbar})$ overnight, resulting in $\mathrm{pDTT}\left(\mathrm{M}_{\mathrm{n}, \mathrm{NMR}}=3000\right.$ $\left.\mathrm{kDa}, \mathrm{M}_{\mathrm{n}, \mathrm{SEC}}=3533 \mathrm{kDa}, Ð=1.34\right)$ as a white powder $(3.52 \mathrm{~g}, 66 \%$ yield $)$.

End-cap replacement in pDTT using 2-mercaptoethanol

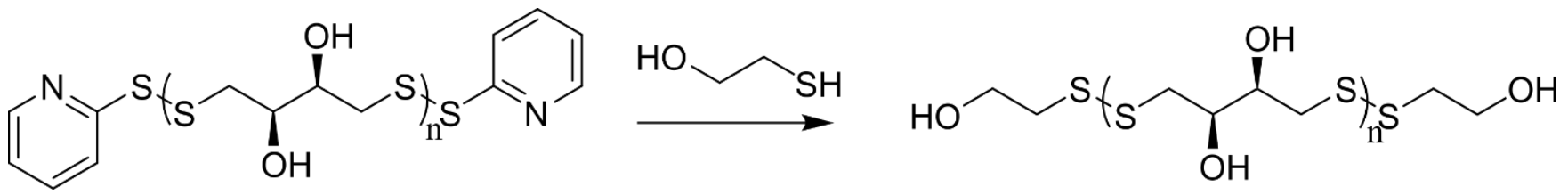

$\mathrm{pDTT}_{20}\left(5.5 \mathrm{mg}, 1.08 \mu \mathrm{mol}\right.$ using $\left.M_{\mathrm{n}, \mathrm{NMR}}=5100 \mathrm{Da}\right)$ was dissolved in $100 \mu \mathrm{L}$ DMSO- $d_{6}$ in a $2 \mathrm{ml}$ vial. A stock solution of 2-mercaptoethanol $(4.0 \mu \mathrm{L}, 56.8 \mu \mathrm{mol})$ was dissolved in $0.20 \mathrm{ml} \mathrm{DMSO}-d_{6}$ in a $2 \mathrm{ml}$ vial, from which $8.5 \mu \mathrm{L}$ ( 2 equiv.) was added to the $\mathrm{pDTT}_{20}$ solution. The reaction mixture was shaken for $1 \mathrm{~h}$. The end group of pDTT was replaced by 2-mercaptoethanol ( $84 \%$ efficiency) without inducing any main chain depolymerization according to ${ }^{1} \mathrm{H}-\mathrm{NMR}$ (Figure S7).

Quantification of cDTT using ${ }^{l} H-N M R$

In general, ${ }^{1} \mathrm{H}-\mathrm{NMR}$ was used to determine the percentage of cDTT formed from the integral of the -OH peak of cDTT $(5.22 \mathrm{ppm})$ divided by the sum of the integrals of this peak and the -OH peak of the linear pDTT (4.91 ppm). Similarly, integration was done on the $-\mathrm{CH}_{2}-\mathrm{O}$ peak of cDTT $(3.03 \mathrm{ppm})$ along with the $-\mathrm{CH}_{2}-\mathrm{O}$ peak of the linear pDTT (2.91 ppm) (e.g., see Figure 2).

\section{Depolymerization of pDTT in presence of DTT}

$\mathrm{pDTT}_{20}\left(30.0 \mathrm{mg}, 5.88 \mu \mathrm{mol}\right.$ using $\left.M_{\mathrm{n}, \mathrm{NMR}}=5100 \mathrm{Da}\right)$ was dissolved in $3.50 \mathrm{ml}$ DMSO- $d_{6}$ in a scintillation vial, equipped with a magnetic stir bar. Three stock solutions were prepared, i.e. (A) $\mathrm{Et}_{3} \mathrm{~N}(5.0 \mu \mathrm{l}, 35.9 \mu \mathrm{mol})$ in 50.0 $\mu \mathrm{l}$ DMSO- $d_{6}$, (B) DTT $(5.0 \mathrm{mg}, 32.4 \mu \mathrm{mol})$ in $50.0 \mu \mathrm{l}$ DMSO- $d_{6}$ and (C) DTDP $(5.2 \mathrm{mg}, 23.5 \mu \mathrm{mol})$ in $0.70 \mathrm{ml}$ DMSO- $d_{6}$. Solution $\mathrm{C}$ was divided into seven small vials. To initiate the depolymerization process, $6.4 \mu \mathrm{A}$ and $14.0 \mu \mathrm{l} \mathrm{B}$ were added at the same time to the $\mathrm{pDTT}_{20}$ solution. Aliquots $(0.50 \mathrm{ml})$ were taken periodically and immediately transferred to one of the seven vials containing $0.1 \mathrm{ml}$ solution $\mathrm{C}$ to quench the depolymerization using DTDP. ${ }^{1} \mathrm{H}-\mathrm{NMR}$ and SEC (identical protocol but with DMF replacing DMSO- $d_{6}$ as solvent) were used to analyze the quenched solutions. In addition, the depolymerization of pDTT was monitored in the absence of $\mathrm{Et}_{3} \mathrm{~N}$, and with and without $\mathrm{N}_{2}$ atmosphere, using the same procedure (Figures 2, 3, S5, S6, S10, S14, S15, and S16).

Degradation of pDTT in basic, acidic, and neutral medium

$\mathrm{pDTT}_{20}\left(24.0 \mathrm{mg}, 4.71 \mu \mathrm{mol}\right.$ using $\left.M_{\mathrm{n}, \mathrm{NMR}}=5100 \mathrm{Da}\right)$ was dissolved in $1.80 \mathrm{ml}$ DMSO- $d_{6}$ and equally divided into three NMR tubes. Two stock solutions were prepared: (A) $\mathrm{Et}_{3} \mathrm{~N}(5.0 \mu 1,35.9 \mu \mathrm{mol})$ in $0.50 \mathrm{ml} \mathrm{DMSO}-d_{6}$ and (B) acetic acid $(5.0 \mu 1,87.4 \mu \mathrm{mol})$ in $0.50 \mathrm{ml}$ DMSO- $d_{6}$. The three NMR tubes were now prepared as follows: (1) 
Supporting Information - Synthesis and Closed-Loop Recycling of Self-Immolative Poly(Dithiothreitol)

$17.0 \mu \mathrm{l} \mathrm{A}\left(1\right.$ equiv. $\mathrm{Et}_{3} \mathrm{~N}$ ), (2) $7.0 \mu \mathrm{l} \mathrm{B}$ (1 equiv. acetic acid), and (3) nothing (blank). All tubes were stored at room temperature under a protective atmosphere of $\mathrm{N}_{2}$ while recording ${ }^{1} \mathrm{H}-\mathrm{NMR}$ spectra periodically (Figure S17).

Thermal stability of pDTT in solution

A ${ }^{1} \mathrm{H}-\mathrm{NMR}$ spectrum was recorded of $\mathrm{pDTT}_{20}\left(8.0 \mathrm{mg}, 1.57 \mu \mathrm{mol}\right.$ using $\left.M_{\mathrm{n}, \mathrm{NMR}}=5100 \mathrm{Da}\right)$ in $0.60 \mathrm{ml}$ DMSO- $d_{6}$. The NMR tube was immerged into an oil bath maintained at $60{ }^{\circ} \mathrm{C} .{ }^{1} \mathrm{H}-\mathrm{NMR}$ spectra were recorded periodically over the next 134 days (Figures S17 and S4). The formation of cDTT is suggested to occur by a disulfide metathesis process (Scheme S1, 1).

\section{Scheme S1. 1) Disulfide Metathesis Expelling cDTT Upon Heating and 2) Homolytic Cleavage of Disulfide at End-Caps After Exposure to UV light.}

1)<smiles>CCSCCC(C)C</smiles>

2)<smiles>O[C@H]1CSSC[C@@H]1O</smiles>

\section{UV-light stability of pDTT in solution}

An ${ }^{1} \mathrm{H}-\mathrm{NMR}$ spectrum was recorded of $\mathrm{pDTT}_{20}\left(7.6 \mathrm{mg}, 1.49 \mu \mathrm{mol}\right.$ using $\left.M_{\mathrm{n}, \mathrm{NMR}}=5100 \mathrm{Da}\right)$ in $0.60 \mathrm{ml}$ DMSO$d_{6}$. The NMR tube was irradiated with UV light $\left(\lambda_{\max }=365 \mathrm{~nm}\right)$ for $1 \mathrm{~h}$. The formation of cDTT is suggested to occur from absorption of UV light by the end-cap resulting in a homolytic cleavage of the disulfide bond at the end-cap (Schemes S1 and S2). The polymer is subsequently reduced to cDTT through radical pathways. After 1 hour exposure to UV light the amount of cDTT was quantified through ${ }^{1} \mathrm{H}-\mathrm{NMR}$ spectroscopy. The tubes was set in a dark cupboard for another hour, and another ${ }^{1} \mathrm{H}-\mathrm{NMR}$ was measured, before subjecting the tube to UV light for another hour. In total, this procedure of on/off irradiation was conducted for $12 \mathrm{~h}$ (Figure S18).

Shelf storage stability of pDTT

${ }^{1} \mathrm{H}-\mathrm{NMR}$ spectra were recorded of $\mathrm{pDTT}_{20}\left(7.6 \mathrm{mg}, 1.49 \mu \mathrm{mol}\right.$ using $\left.M_{\mathrm{n}, \mathrm{NMR}}=5100 \mathrm{Da}\right)$ in DMSO- $d_{6}$ before and after storage at room temperature for 270 days. No changes to the polymer structure was found (Figure S19).

Thermal stability of pDTT in solid state

$\mathrm{pDTT}_{20}\left(0.100 \mathrm{~g}, 19.6 \mu \mathrm{mol}\right.$ using $\left.M_{\mathrm{n}, \mathrm{NMR}}=5100 \mathrm{Da}\right)$ was added to a $10 \mathrm{ml}$ round bottom flask. A ${ }^{1} \mathrm{H}-\mathrm{NMR}$ spectrum was recorded of a $\sim 10 \mathrm{mg}$ sample (taken from the $\mathrm{pDTT}_{20}$ lump) in $0.60 \mathrm{ml}$ DMSO- $d_{6}$. The flask was immerged into an oil bath maintained at $60{ }^{\circ} \mathrm{C}$. ${ }^{1} \mathrm{H}-\mathrm{NMR}$ spectra were recorded periodically over the next 35 days by taking $\sim 10 \mathrm{mg}$ pDTT samples from the flask and dissolve it in $0.6 \mathrm{ml} \mathrm{DMSO}-d_{6}$ in an NMR tube (Figure S4). The formation of cDTT is suggested to occur by a disulfide metathesis process (Scheme S1). 
Supporting Information - Synthesis and Closed-Loop Recycling of Self-Immolative Poly(Dithiothreitol)

(I) ${ }^{l} \mathrm{H}$-NMR recording of the solid-state depolymerization of pDTT (undissolved in $\mathrm{D}_{2} \mathrm{O}$ ) in the presence of $D T T / E t_{3} N$

Ambient conditions: Solid pDTT 20 was cut into 2 pieces $\left(13.0 \mathrm{mg} / \mathrm{pcs}, 4.33 \mu \mathrm{mol} / \mathrm{pcs}\right.$ using $\left.M_{\mathrm{n}, \mathrm{NMR}}=3000 \mathrm{Da}\right)$, each placed in an NMR tube of its own. Two stock solutions were prepared: (A) DTT (13.5 mg, $86 \mu \mathrm{mol})$ in 0.50 $\mathrm{ml} \mathrm{D}_{2} \mathrm{O}$ and $(\mathrm{B}) \mathrm{Et}_{3} \mathrm{~N}(12.0 \mu \mathrm{l}, 86.0 \mu \mathrm{mol})$ in $1.00 \mathrm{ml} \mathrm{D}_{2} \mathrm{O}$. Tube 1 was added $0.6 \mathrm{~mL} \mathrm{D}_{2} \mathrm{O}$. Tube 2 was added 0.4 $\mathrm{mL} \mathrm{D}_{2} \mathrm{O}$ along with $0.1 \mathrm{~mL} \mathrm{~A}$ and $0.1 \mathrm{~mL} \mathrm{~B}$. All tubes were stored at room temperature sealed with a standard NMR lid. ${ }^{1} \mathrm{H}-\mathrm{NMR}$ and visual inspections were used to follow the depolymerization of pDTT (Figure 5; see also the spectrum of pyridine-2-thiol in Figure S20 and cDTT Figure S21).

Inert conditions: Solid pDTT ${ }_{20}$ was cut into 4 pieces $\left(8.0 \mathrm{mg} / \mathrm{pcs}, 1.57 \mu \mathrm{mol} / \mathrm{pcs}\right.$ using $\left.M_{\mathrm{n}, \mathrm{NMR}}=5100 \mathrm{Da}\right)$, each placed in an NMR tube of its own containing $0.60 \mathrm{ml} \mathrm{D}_{2} \mathrm{O}$ (pre-purged with $\mathrm{N}_{2}$ ). Two stock solutions were prepared: (A) DTT $(1.5 \mathrm{mg}, 9.73 \mu \mathrm{mol})$ in $0.10 \mathrm{ml} \mathrm{D}_{2} \mathrm{O}$ and (B) $\mathrm{Et}_{3} \mathrm{~N}(5.0 \mu 1,35.9 \mu \mathrm{mol})$ in $0.50 \mathrm{ml} \mathrm{D}_{2} \mathrm{O}$. The four NMR tubes were prepared as follows: (1) $33.0 \mu \mathrm{l}$ of A (2 equiv. DTT) and $17.0 \mu \mathrm{l} \mathrm{B} \mathrm{(1} \mathrm{equiv.} \mathrm{Et}{ }_{3} \mathrm{~N}$ ), (2) $33.0 \mu \mathrm{l}$ A (2 equiv. DTT), (3) $17.0 \mu 1 \mathrm{~B}\left(1\right.$ equiv. $\mathrm{Et}_{3} \mathrm{~N}$ ) and (4) nothing (blank). All tubes were stored at room temperature under a protective atmosphere of $\mathrm{N}_{2} .{ }^{1} \mathrm{H}-\mathrm{NMR}$ and visual inspections were used to follow the depolymerization of pDTT (Figure S7; see also the spectrum of pyridine-2-thiol in Figure S20 and cDTT Figure S21).

(II) Video recording of the solid-state depolymerization of pDTT (undissolved in $\mathrm{D}_{2} \mathrm{O}$ ) in the presence of DTT/Et ${ }_{3} \mathrm{~N}$ Solid pDTT 20 was cut into two square pieces $(14.0 \mathrm{mg} / \mathrm{pcs})$, each placed in a small petri dish of its own. The petri dishes with a small stir bar were placed in a glass chamber maintaining a constant flux of $\mathrm{N}_{2}$ throughout the experiment. The first petri dish was used as a reference, in that nothing else but $4 \mathrm{ml}$ deionized water pre-purged with $\mathrm{N}_{2}$ was added. To the second petri dish, $2.88 \mathrm{ml}$ deionized water was added along with DTT (3.6 mg dissolved in $1.00 \mathrm{ml}$ deionized water $)$ and $\mathrm{Et}_{3} \mathrm{~N}\left(0.120 \mathrm{ml}\right.$ from a stock solution containing $5.0 \mu \mathrm{Et}_{3} \mathrm{~N}$ in $1.00 \mathrm{ml}$ deionized water) to reach a total volume of $4 \mathrm{ml}$. The depolymerization was recorded using a camera taking pictures every second minute. The reaction setup was covered by a cardboard box to optimize the light recording conditions. The video material includes a timelapse of the photo series of the first $180 \mathrm{~min}$ of the depolymerization, shown at five frames $\mathrm{s}^{-1}$. 
Recycling protocol

Scheme S2. Reaction Protocol Describing the Recycling Pathway of pDTT.

S1)

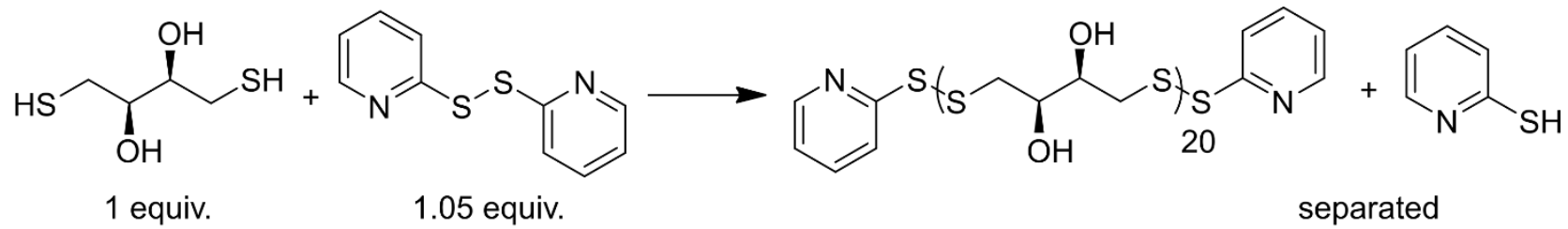

S2)

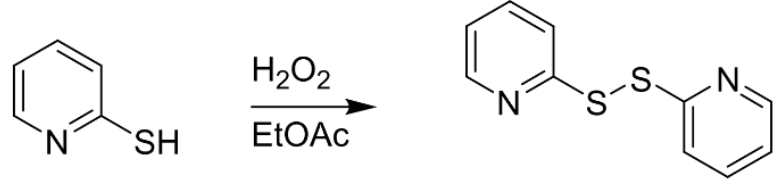

S3)

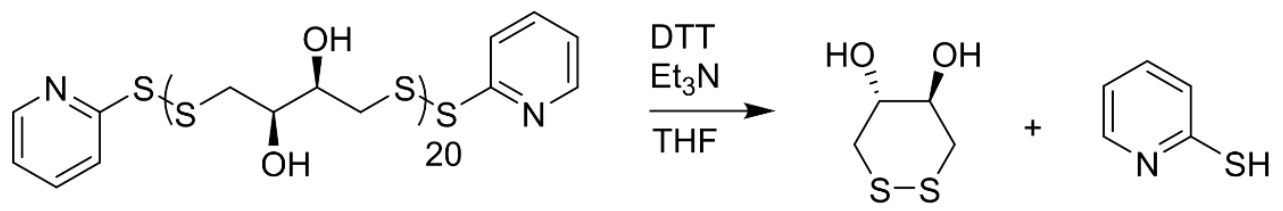

S4)

S5)
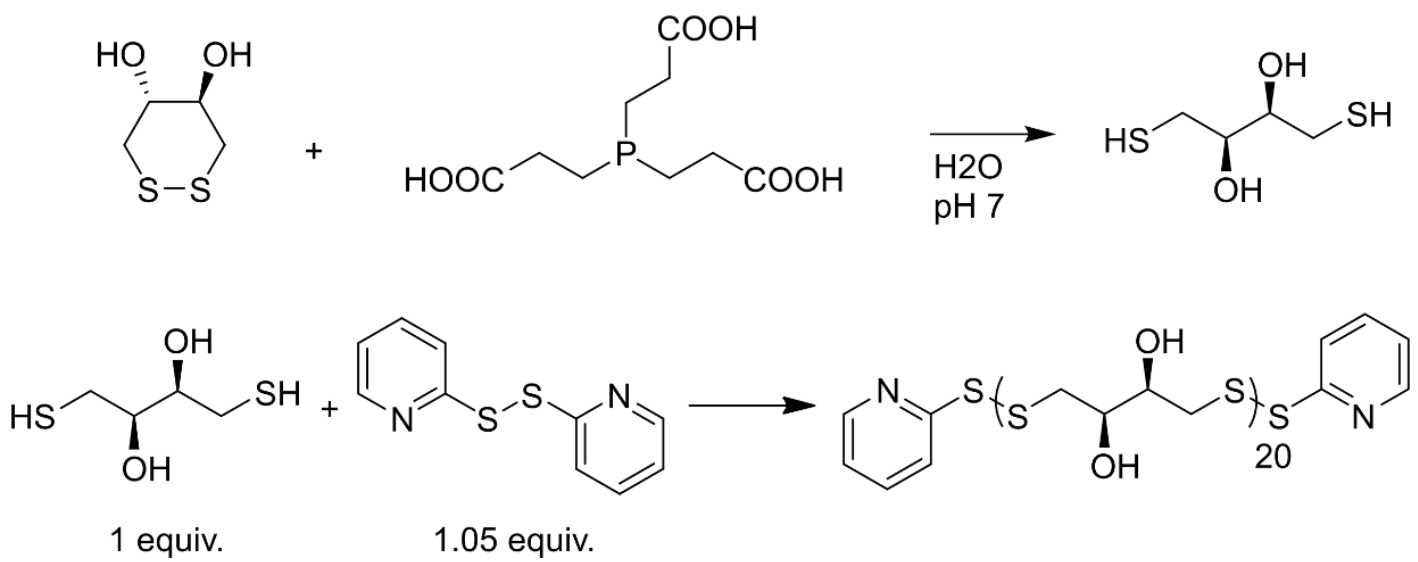

Recycling of pDTT could be accomplished through five steps (see Scheme S2):

(S1) Polymerization. DTT (1.00 g, $6.48 \mathrm{mmol})$ and DTDP $(1.50 \mathrm{~g}, 6.81 \mathrm{mmol}, \xi=0.05)$ were polymerized according to the standard protocol (vide supra), providing pDTT $20(0.70 \mathrm{~g}, 69 \%)$ with $M_{\mathrm{n}, \mathrm{SEC}}=11200 \mathrm{Da}$ and $Ð=$ 1.32. ${ }^{1} \mathrm{H}-\mathrm{NMR}$ DMSO- $d_{6}(400 \mathrm{MHz}) \delta=8.46(\mathrm{~d}, 1 \mathrm{H}, J=4.9 \mathrm{~Hz}), 7.82(\mathrm{~m}, 2 \mathrm{H}), 7.25(\mathrm{t}, 1 \mathrm{H}, J=6.5 \mathrm{~Hz}), 5.16(\mathrm{~d}$, $1 \mathrm{H}, J=6.2 \mathrm{~Hz}), 4.99(\mathrm{~d}, 1 \mathrm{H}, J=6.2 \mathrm{~Hz}), 4.91(\mathrm{~d}, 46 \mathrm{H}, J=6.3 \mathrm{~Hz}), 3.72(\mathrm{~s}, 47 \mathrm{H}), 2.91(\mathrm{dd}, 50 \mathrm{H}, J=4.4 \mathrm{~Hz}, J=$ $13.2 \mathrm{~Hz}), 2.78(\mathrm{~m}, 50 \mathrm{H})$. During the purification of $\mathrm{pDTT}_{20}$ [by precipitation from DMF into $\mathrm{CHCl}_{3} /$ hexane $(1: 1$ $\mathrm{v} / \mathrm{v})$ ], the organic precipitate, containing mostly pyridine-2-thiol, was isolated and reduced in vacuo. ${ }^{1} \mathrm{H}-\mathrm{NMR}$ $\mathrm{CDCl}_{3}(400 \mathrm{MHz}) \delta=14.0(\mathrm{~s}, 1 \mathrm{H}), 7.58(\mathrm{~d}, 1 \mathrm{H}, J=6.0 \mathrm{~Hz}), 7.54(\mathrm{~d}, 1 \mathrm{H}, J=8.7 \mathrm{~Hz}), 7.38(\mathrm{t}, 1 \mathrm{H}, J=7.3 \mathrm{~Hz})$, $6.77(\mathrm{t}, 1 \mathrm{H}, J=6.5 \mathrm{~Hz})$.

(S2) Recovery of DTDP. The isolated pyridine-2-thiol from the first step was dissolved in $6 \mathrm{ml}$ EtOAc in a $25 \mathrm{ml}$ round bottom flask equipped with a magnetic stir bar, and cooled to $0{ }^{\circ} \mathrm{C}$ using an ice bath. The solution was oxidized using $0.63 \mathrm{ml} 30 \% \mathrm{H}_{2} \mathrm{O}_{2}$ in water added dropwise over $15 \mathrm{~min}$. The reaction was left to proceed at $0{ }^{\circ} \mathrm{C}$ 
Supporting Information - Synthesis and Closed-Loop Recycling of Self-Immolative Poly(Dithiothreitol)

for $30 \mathrm{~min}$, and then at room temperature for additional $2 \mathrm{~h}$. Next, $20 \mathrm{ml}$ demineralized water was added and the reaction was transferred to a separatory funnel. The water phase was extracted twice with $20 \mathrm{ml}$ EtOAc, the combined organic phases were dried over $\mathrm{MgSO}_{4}$ and reduced in vacuo. The crude product was purified using silicate column chromatography [EtOAc/hexane $(3: 7 \mathrm{v} / \mathrm{v})$, silicate pore size: $60 \AA$ ] producing 2,2'-dithiodipyridine $(1.17 \mathrm{~g}, 78 \%) .{ }^{1} \mathrm{H}-\mathrm{NMR} \mathrm{CDCl}_{3}(400 \mathrm{MHz}) \delta=8.46(\mathrm{~d}, 1 \mathrm{H}, J=4.7 \mathrm{~Hz}), 7.62(\mathrm{~m}, 2 \mathrm{H}), 7.11(\mathrm{~m}, 1 \mathrm{H})$.

(S3) SIP depolymerization of pDTT. pDTT 20 obtained in the first step $(0.700 \mathrm{~g}, 94.0 \mu \mathrm{mol})$ was partially dissolved in $5 \mathrm{ml}$ THF in a $25 \mathrm{ml}$ round bottom flask equipped with a magnetic stir bar. DTT $(29.0 \mathrm{mg}, 0.188 \mathrm{mmol})$ was added, turning the solution yellow, followed by addition of $\mathrm{Et}_{3} \mathrm{~N}(13.0 \mu \mathrm{L}, 94.0 \mu \mathrm{mol})$. This dissolved the polymers within $2 \mathrm{~min}$. The reaction was left to proceed for $15 \mathrm{~min}$ and the THF removed in vacuo. The cDTT generated in the depolymerization was recrystallized from EtOAc/pentane $(1: 2 \mathrm{v} / \mathrm{v})$ and isolated as a white powder through filtration $(0.442 \mathrm{~g}, 61 \%) .{ }^{1} \mathrm{H}-\mathrm{NMR}$ DMSO- $d_{6}(400 \mathrm{MHz}) \delta=5.22(\mathrm{~s}, 2 \mathrm{H}), 3.34(\mathrm{~d}, 2 \mathrm{H}, J=8.5 \mathrm{~Hz}), 3.03(\mathrm{~d}, 2 \mathrm{H}, J$ $=13.0 \mathrm{~Hz}), 2.73(\mathrm{dd}, 2 \mathrm{H}, J=9.5 \mathrm{~Hz}, J=12.8 \mathrm{~Hz})$.

(S4) Recovery of DTT. The cDTT produced in the third step was ring-opened using tris(2-carboxyethyl)phosphine (TCEP) as reductant. In a $100 \mathrm{ml}$ round bottom flask equipped with a magnetic stir bar, cDTT (0.400 g, 2.59 $\mathrm{mmol})$ and TCEP $(0.818 \mathrm{~g}, 2.85 \mathrm{mmol})$ were dissolved in $40 \mathrm{ml} 0.1 \mathrm{M}$ phosphate buffered water $(\mathrm{pH} 7)$. The reaction was stirred at room temperature for $2 \mathrm{~h}$, transferred to a separatory funnel and extracted 8 times with 20 $\mathrm{ml}$ dichloromethane. The dichloromethane was removed in vacuo to give DTT $(0.284 \mathrm{~g}, 72 \%)$. ${ }^{1} \mathrm{H}-\mathrm{NMR}^{\mathrm{CDCl}} \mathrm{CD}_{3}$ (400 MHz) $\delta=3.67(\mathrm{~m}, 2 \mathrm{H}), 2.81-2.66(\mathrm{~m}, 4 \mathrm{H}), 2.51(\mathrm{~s}, 2 \mathrm{H}), 1.50(\mathrm{t}, 2 \mathrm{H}, J=8.6 \mathrm{~Hz})$.

(S5) Repolymerization of recovered DTT and DTDP. Recovered DTT $(0.284 \mathrm{~g}, 1.84 \mathrm{mmol})$ from the fourth step and DTDP $(0.426 \mathrm{~g}, 1.93 \mathrm{mmol}, \xi=0.05)$ from the second step were polymerized according to the standard protocol (vide supra), providing $\mathrm{pDTT}_{20}\left(0.23 \mathrm{~g}, 76 \%\right.$ ) with $M_{\mathrm{n}, \mathrm{SEC}}=7100 \mathrm{Da}$ and $\doteq=1.45 .{ }^{1} \mathrm{H}-\mathrm{NMR} \mathrm{DMSO}-d_{6}$ $(400 \mathrm{MHz}) \delta=8.46(\mathrm{~d}, 1 \mathrm{H}, J=4.7 \mathrm{~Hz}), 7.82(\mathrm{~m}, 2 \mathrm{H}), 7.24(\mathrm{t}, 1 \mathrm{H}, J=6.5 \mathrm{~Hz}), 5.16(\mathrm{~d}, 1 \mathrm{H}, J=6.3 \mathrm{~Hz}), 4.98(\mathrm{~d}$, $1 \mathrm{H}, J=6.3 \mathrm{~Hz}) 4.92(\mathrm{~d}, 38 \mathrm{H}, J=6.2 \mathrm{~Hz}), 3.72(\mathrm{~s}, 37 \mathrm{H}), 2.93(\mathrm{dd}, 40 \mathrm{H}, J=4.4 \mathrm{~Hz}, J=13.2 \mathrm{~Hz}), 2.78(\mathrm{~m}, 40 \mathrm{H})$

\section{$T G A$}

Samples of $\sim 2 \mathrm{mg}$ pDTT 20 were loaded into $\mathrm{Al}_{2} \mathrm{O}_{3}$ pans (volume $=50 \mu \mathrm{L}$ ) and heated from $25-500{ }^{\circ} \mathrm{C}$ using $10{ }^{\circ} \mathrm{C}$ $\mathrm{min}^{-1}$ as gradient in a $\mathrm{N}_{2}$ atmosphere (flow $=20 \mathrm{ml} \mathrm{min}^{-1}$ ) (Figure S22).

DSC

Samples of $\sim 11 \mathrm{mg}$ pDTT 20 were loaded into $\mathrm{Al}_{2} \mathrm{O}_{5}$ pans (volume $=50 \mu \mathrm{L}$ ) and rapidly cooled down to $-25{ }^{\circ} \mathrm{C}$ in a $\mathrm{N}_{2}$ atmosphere. The glass transition temperature $\left(T_{\mathrm{g}}\right)$ was measured using modulated temperature differential scanning calorimetry (MTDSC) in the temperature range from $0-100{ }^{\circ} \mathrm{C}$ using $5{ }^{\circ} \mathrm{C} \mathrm{min}^{-1}$ as gradient (in steps of $1{ }^{\circ} \mathrm{C}$ ), until thermal equilibrium was reached as evidenced from a constant heat flow. Prior to this measurement the solvent residues were stripped at $100{ }^{\circ} \mathrm{C}$ for $20 \mathrm{~min}$. The specific heat was calculated as the integration of the heat flow of each individual peak over time baselined with respect to the isothermal plateau. Finally, the $T_{\mathrm{g}}$ (= $44{ }^{\circ} \mathrm{C}$ ) was calculated by using the half-cp method with respect to the specific heat data (Figure S23).

\section{$G P C$}

Samples of $\sim 3.0 \mathrm{mg}$ pDTT (mass corrected for the contributions from, typically, DMF and chloroform residues determined by ${ }^{1} \mathrm{H}-\mathrm{NMR}$ ) were dissolved in $1 \mathrm{ml}$ DMF containing $10 \mathrm{mM} \mathrm{LiBr}$. The value of $\mathrm{dn} / \mathrm{dc}$ was acquired using a $100 \%$ mass recovery technique. Figures S24-S28 display the traces for the samples of pDTT $10, \mathrm{pDTT}_{20}$, $\mathrm{pDTT}_{40}, \mathrm{pDTT}_{100}$ and $\mathrm{pDTT}_{200}$, respectively, obtained by means of size exclusion chromatography using a multiangle laser light scattering (SEC-MALLS) detector. In accordance with expectation, the elution volume 
Supporting Information - Synthesis and Closed-Loop Recycling of Self-Immolative Poly(Dithiothreitol)

comes earlier for the larger polymers. From such experiments the molecular weight $\left(M_{\mathrm{n}, \mathrm{SEC}}\right)$ and the dispersity $(\nexists)$ were extracted along with $\mathrm{DP}_{\mathrm{SEC}}$ (using a rearranged eq. 2).

\section{Physical Properties of pDTT}

All pDTTs are easily soluble in DMF and DMSO, and in THF if DP $\leq 40$, but not $\mathrm{H}_{2} \mathrm{O}$, even after heating or ultrasonication. The insolubility in $\mathrm{H}_{2} \mathrm{O}$ is attributed to the presence of extensive internal hydrogen bonding as seen in e.g. cellulose. The polymers have a high stability in the solid state showing no signs of degradation after storage for 270 days at ambient conditions (Figure S19). At a $60{ }^{\circ} \mathrm{C}$ storage temperature, the degradation of $\mathrm{pDTT}_{20}$ amounts to $4 \%$ after 4 days, identified by ${ }^{1} \mathrm{H}-\mathrm{NMR}$, but with no further breakdown thereafter as tested over the next month (Figure S4). TGA reveals a minor loss of weight $\left(5.6 \%\right.$ ) for $\mathrm{pDTT}_{20}$ until $100{ }^{\circ} \mathrm{C}$ (Figure S22) due to the evaporation of solvent residues (THF, chloroform and water) identified by ${ }^{1} \mathrm{H}-\mathrm{NMR}$ spectroscopy (Figure S29). Differential thermogravimetry reveals a peak at $213{ }^{\circ} \mathrm{C}$ with the decomposition initiating at $\sim 200{ }^{\circ} \mathrm{C}$. The value of $T_{\mathrm{g}}$ of $\mathrm{pDTT}_{20}$ is measured by MTDSC on the basis of the half-cp method to be $44{ }^{\circ} \mathrm{C}$ (Figure S23). The absence of a second exothermal peak is consistent with a lack of crystallinity in pDTT 20.

Thermal, photochemical, and chemical stability of pDTT in solution

Figure S30 shows the ${ }^{1} \mathrm{H}-\mathrm{NMR}$ spectra of $\mathrm{pDTT}_{20}$ in DMSO- $d_{6}$ heated to $60{ }^{\circ} \mathrm{C}$. As seen, the pyridine disulfide end-cap remains in place as deduced from the lack of a pyridine-2-thiol signal at $\delta=6.75 \mathrm{ppm}$. Instead, DTDP is formed with the characteristic signals appearing at $\delta=8.48,7.80,7.63$, and 7.28 ppm (see Figure S10 for comparison). Along with this, cDTT is generated $(\delta=5.22 \mathrm{ppm})$, at the expense of pDTT ( $\delta=4.91 \mathrm{ppm})$, in $\sim 15 \%$ yield after 7 days and in almost $100 \%$ yield after 130 days at $60{ }^{\circ} \mathrm{C}$ (Figure S17). Knowing that the SIP degradation mechanism cannot be the one in play since it would be much faster, the cDTT is suggested to be formed in intramolecular disulfide metathesis reactions (Scheme S1). It is well documented that disulfide bonds can be susceptible to disulfide exchange/metathesis in solutions if exposed to heating ${ }^{\mathrm{S} 1, \mathrm{~S} 2}$ or UV light. ${ }^{\mathrm{S} 3, \mathrm{~S} 4}$ In fact, UV radiation at $365 \mathrm{~nm}$ of the $\mathrm{pDTT}_{20}$ in DMSO- $d_{6}$ leads to $40 \%$ degradation in $1 \mathrm{~h}$ and $90 \%$ in $5 \mathrm{~h}$ with the formation of cDTT (Figure S18). Switching on and off the radiation reveals that the degradation only occurs during exposure to UV.

Concerning the chemical stability, no degradation of $\mathrm{pDTT}_{20}$ is induced by acidic conditions ( $2 \mathrm{mM}$ acetic acid in DMSO- $d_{6}$ ) over a week, although the pyridine disulfide end-cap could be expected to be susceptible to nucleophilic substitution once protonated. Under basic conditions ( $2 \mathrm{mM} \mathrm{Et}_{3} \mathrm{~N}$ in DMSO- $d_{6}$ ), $\mathrm{pDTT}_{20}$ degrades to cDTT (25\%) in $5 \mathrm{~h}$, measured from ${ }^{1} \mathrm{H}-\mathrm{NMR}$. Most likely, the depolymerization proceeds through a nucleophilic addition of $\mathrm{Et}_{3} \mathrm{~N}$ into the disulfide bond in a similar way as phosphine reagents can reduce disulfides. ${ }^{\mathrm{S} 5}$ 
Supporting Information - Synthesis and Closed-Loop Recycling of Self-Immolative Poly(Dithiothreitol)

\section{Polymers}

Table S1 summarizes the most characteristic parameters determined for the samples of $\mathrm{pDTT}_{10}, \mathrm{pDTT}_{20}, \mathrm{pDTT}_{40}$, pDTT $_{100}$ and pDTT $_{200}$ determined by ${ }^{1} \mathrm{H}-\mathrm{NMR}$ and SEC-MALLS. Figures S24-S28 show the SEC traces, while Figures S31-S34 display the ${ }^{1} \mathrm{H}-\mathrm{NMR}$ spectra (pDTT 20 in Figure 2).

Table S1. Monomer Ratios, Yields, and the Characteristic Parameters for the Synthesized Poly(disulfide)s in terms of DP, M, and $Đ$.

\begin{tabular}{llllllllll}
\hline Polymer & DTT: DTDP & $\begin{array}{l}\text { Yield } \\
(\%)\end{array}$ & DP $_{\text {theo }}$ & DP NMR & DPSEC & $\begin{array}{l}M_{\mathrm{n}, \text { theo }} \\
\mathrm{g} \mathrm{mol}^{-1}\end{array}$ & $\begin{array}{l}M_{\mathrm{n}, \mathrm{NMR}} \\
\mathrm{g} \mathrm{mol}^{-1}\end{array}$ & $\begin{array}{l}M_{\mathrm{n}, \mathrm{SEC}} \\
\mathrm{g} \mathrm{mol}^{-1}\end{array}$ & $Ð$ \\
\hline $\operatorname{pDTT}_{10}$ & $1: 1.1$ & 71 & 10 & 18 & 20 & 1740 & 3000 & 3200 & 1.30 \\
$\operatorname{pDTT}_{20}$ & $1: 1.05$ & 83 & 20 & 32 & 33 & 3260 & $\begin{array}{l}5100 \\
( \pm 1040)^{a}\end{array}$ & $\begin{array}{l}5700 \\
( \pm 1300)^{a}\end{array}$ & $\begin{array}{l}1.31 \\
( \pm 0.05)^{a}\end{array}$ \\
$\operatorname{pDTT}_{40}$ & $1: 1.025$ & 69 & 40 & 64 & 69 & 6310 & 10000 & 10700 & 1.26 \\
$\operatorname{pDTT}_{100}$ & $1: 1.01$ & 63 & 100 & 110 & 87 & 15420 & 17100 & 13500 & 1.29 \\
$\operatorname{pDTT}_{200}$ & $1: 1.005$ & 78 & 200 & 93 & 64 & 30620 & 14500 & 10000 & 1.31 \\
\hline
\end{tabular}

${ }^{a}$ Uncertainties were calculated for $\mathrm{pDTT}_{20}$ by synthesizing 3 different samples. 
Supporting Information - Synthesis and Closed-Loop Recycling of Self-Immolative Poly(Dithiothreitol)

\section{Figures}

Comparison of UV and RI signal (SEC)

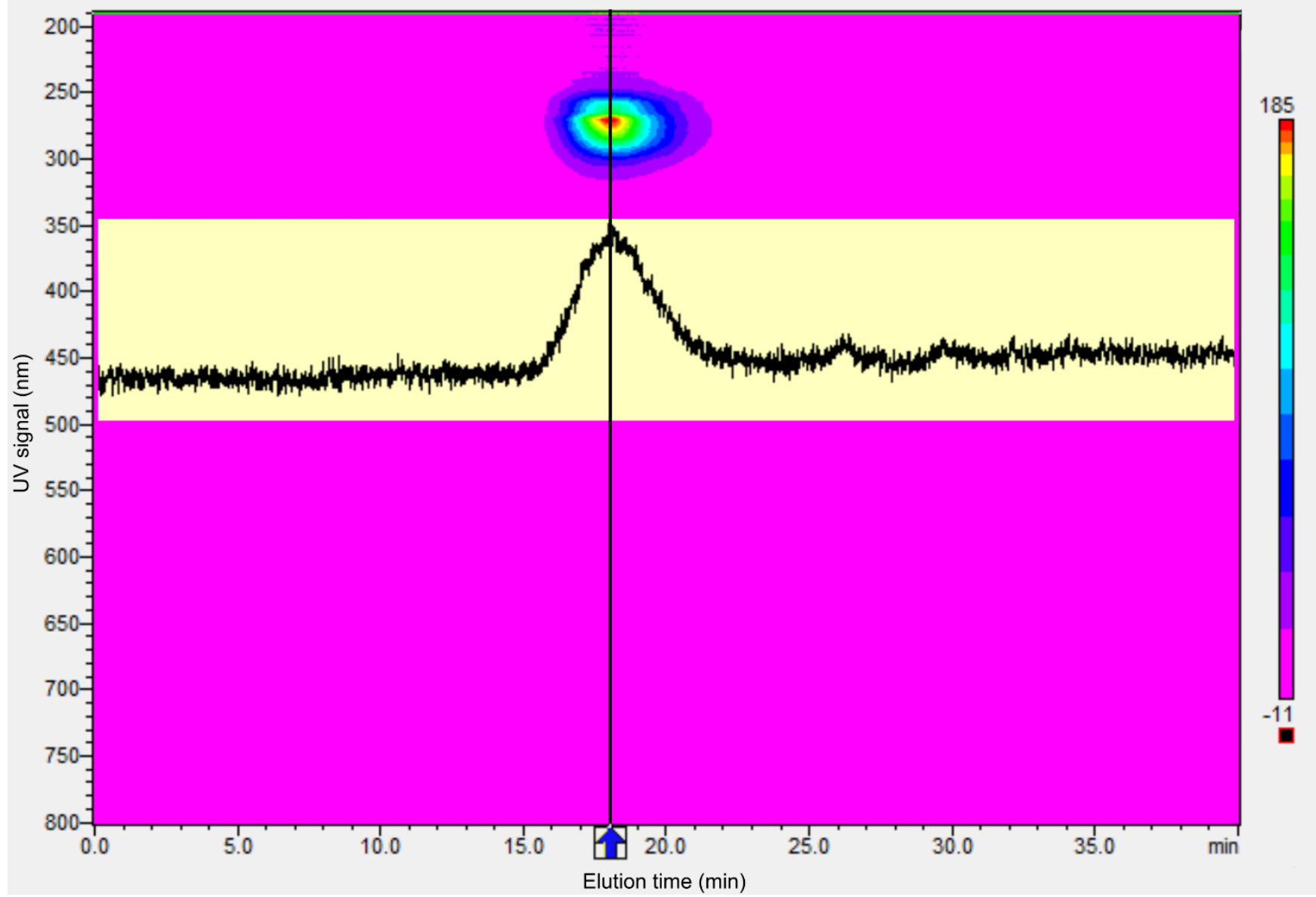

Figure S1. UV detector signals from SEC of $\mathrm{pDTT}_{20}$ for a full SEC chromatogram (40 minutes) at $1 \mathrm{~mL} / \mathrm{min}$. Showing UV signal intensity (color coded) at wavelength to a given elution time. Inset in yellow: RI detector signal from same SEC trace. Black line: max intensity observed for both UV and RI at same elution time. 
Supporting Information - Synthesis and Closed-Loop Recycling of Self-Immolative Poly(Dithiothreitol)

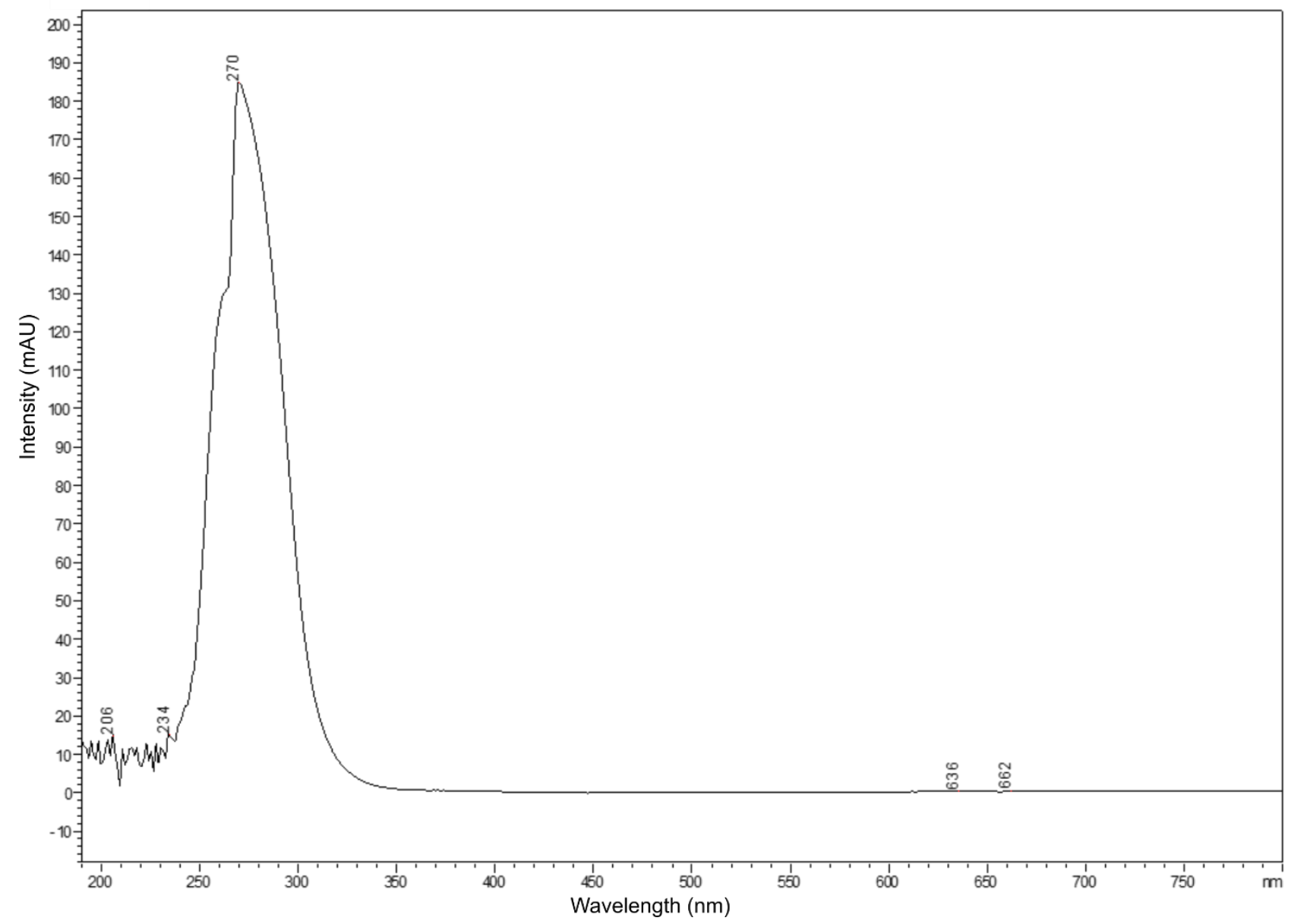

Figure S2. UV/Vis spectrum recorded for SEC of $\mathrm{pDTT}_{20}$ at elution time $22 \mathrm{~min}$. Signal below $268 \mathrm{~nm}$ is disregarded due to DMF's own absorption. 
Supporting Information - Synthesis and Closed-Loop Recycling of Self-Immolative Poly(Dithiothreitol)

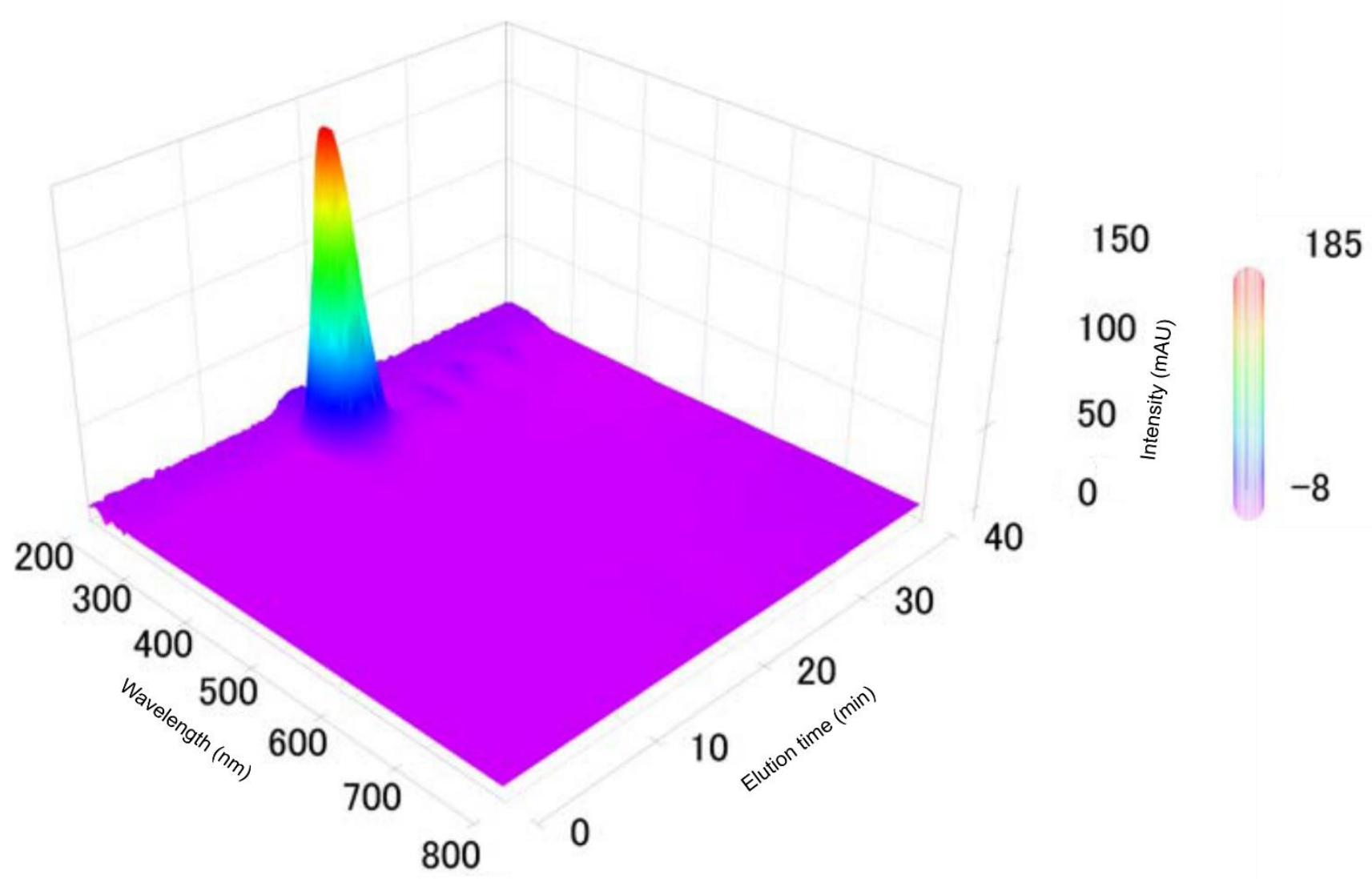

Figure S3. 3-Dimensional representation of figure S1. UV detector signals from SEC of pDTT 20 for a full SEC chromatogram (40 minutes) at $1 \mathrm{~mL} / \mathrm{min}$. Showing UV signal intensity (color coded and z-axis) at wavelength to a given elution time. Signal below $268 \mathrm{~nm}$ is disregarded due to DMF's own absorption. 
Supporting Information - Synthesis and Closed-Loop Recycling of Self-Immolative Poly(Dithiothreitol)

Degradation of pDTT 20 in solid state and solution induced by $60{ }^{\circ} \mathrm{C}$ heat $\left({ }^{1} \mathrm{H}-\mathrm{NMR}\right)$

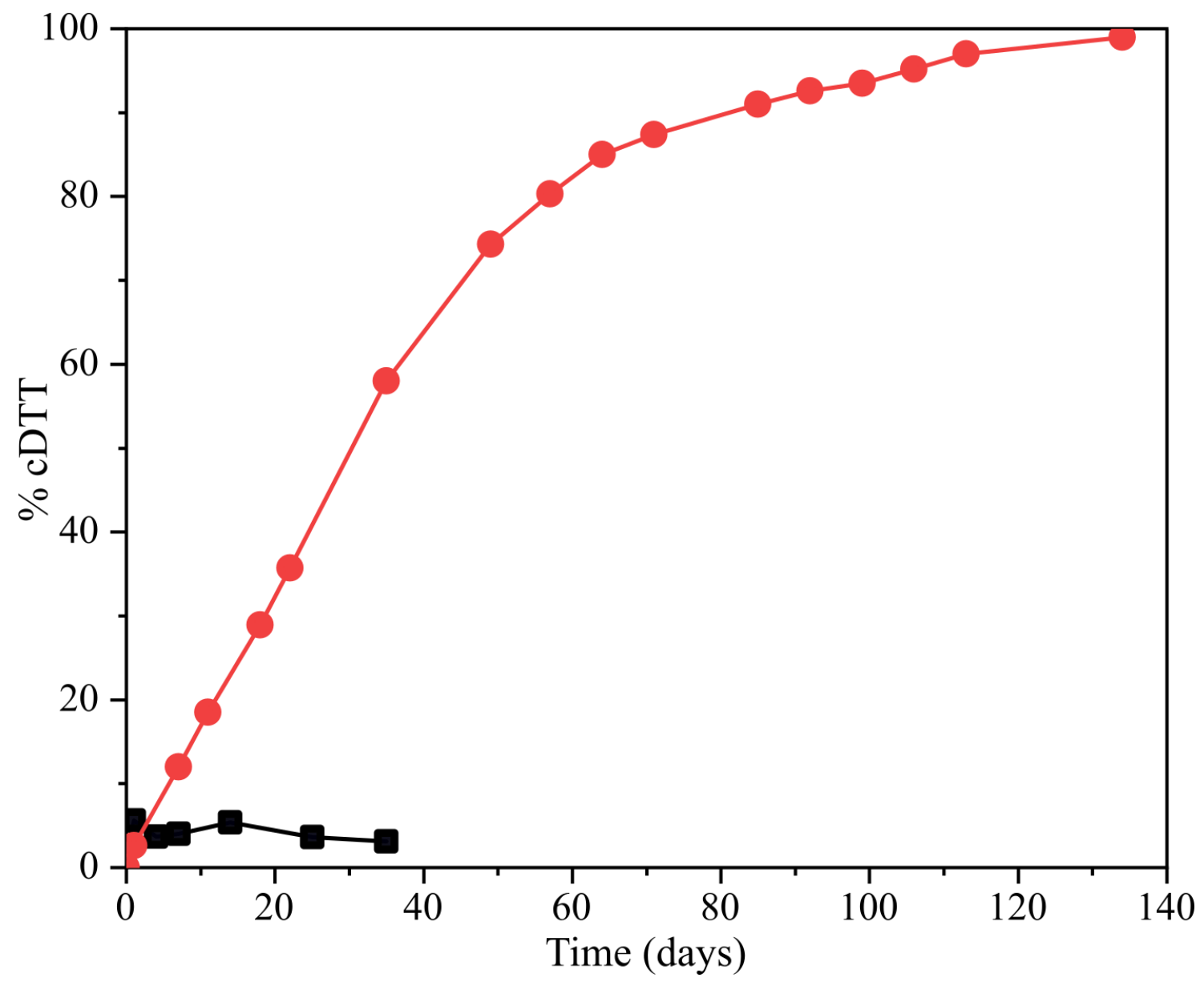

Figure S4. Kinetic traces showing the percentage of cDTT (determined by ${ }^{1} \mathrm{H}-\mathrm{NMR}$ ) formed from pDTT 20 (precipitated from THF) as a function of time in solid state $(\bullet)$ and DMSO- $d_{6}(\bullet)$ at $60^{\circ} \mathrm{C}$. For the solid state measurements, samples $(\sim 10 \mathrm{mg})$ were extracted at specific time intervals and dissolved in DMSO- $d_{6}$ for ${ }^{1} \mathrm{H}-\mathrm{NMR}$ quantification. 
Supporting Information - Synthesis and Closed-Loop Recycling of Self-Immolative Poly(Dithiothreitol)

End-cap exchange using DTT $\left({ }^{1} H-N M R\right)$<smiles>Sc1ccccn1</smiles>

Pyridine-2-thiol

$5 \mathrm{~min}$<smiles>C[Si](C)(C)C[C@H](O)[C@@H](O)C[Si]c1ccccn1</smiles>

p D T T

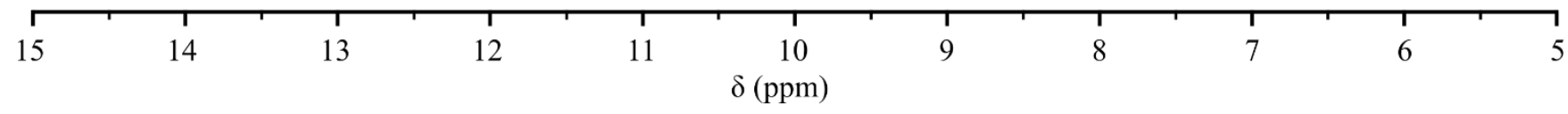

Figure S5. ${ }^{1} \mathrm{H}-\mathrm{NMR}$ spectra of $\mathrm{pDTT}_{20}$ (bottom, black), the depolymerization products obtained 5 min after addition of DTT (middle, green) and pyridine-2-thiol (top, turqoise) serving as reference in DMSO- $d_{6}$ (see complete spectra in Figure S6). 
Supporting Information - Synthesis and Closed-Loop Recycling of Self-Immolative Poly(Dithiothreitol)

End-cap exchange using DTT $\left({ }^{1} H\right.$-NMR $)$-full spectra<smiles>Sc1ccccn1</smiles>

\section{Pyridine-2-thiol}

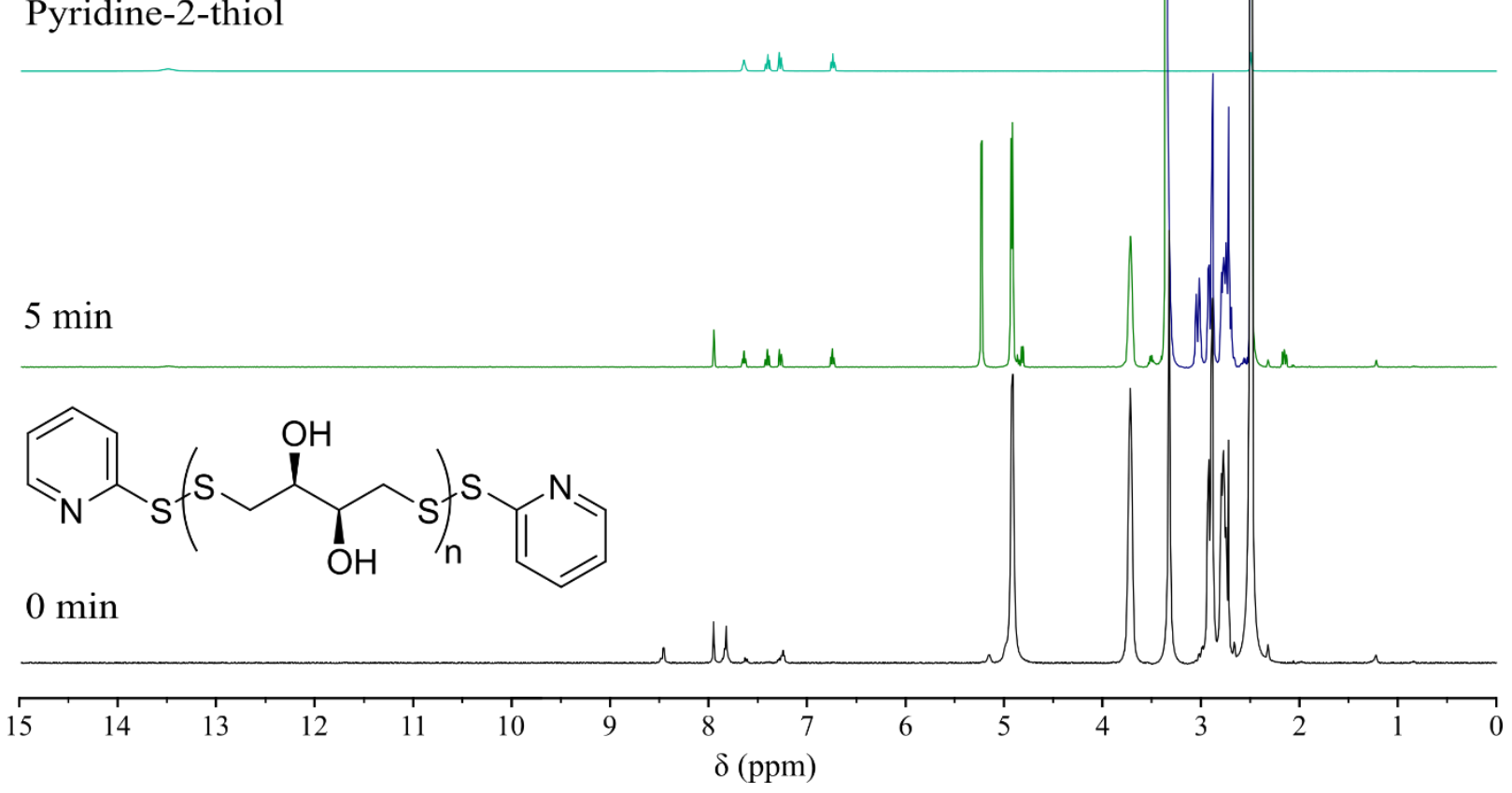

Figure S6. ${ }^{1} \mathrm{H}-\mathrm{NMR}$ spectra of $\mathrm{pDTT}_{20}$ (bottom, black), the depolymerization products obtained 5 min after addition of DTT (middle, green) and pyridine-2-thiol (top, turqoise) serving as reference in DMSO- $d_{6}$. Residues of DMF released from $\mathrm{pDTT}_{20}$ are apparent from the beginning $(\delta=7.92,2.92,2.75 \mathrm{ppm})$, in addition to peaks from DMSO- $d_{6}(2.50 \mathrm{ppm})$ and water (3.33 ppm). 
Supporting Information - Synthesis and Closed-Loop Recycling of Self-Immolative Poly(Dithiothreitol)

End-cap exchange using 2-mercaptoethanol $\left({ }^{1} H\right.$-NMR)

$\left.\mathrm{SO}_{\mathrm{S}}^{\mathrm{HO}} \mathrm{I}_{\mathrm{OH}}^{\mathrm{OH}}\right)_{\mathrm{n}}^{\mathrm{S}} \sim \mathrm{OH}_{\mathrm{OH}}$

pDTT 2-mercaptoethanol end-cap

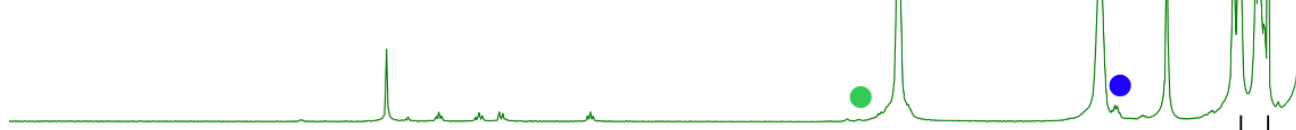

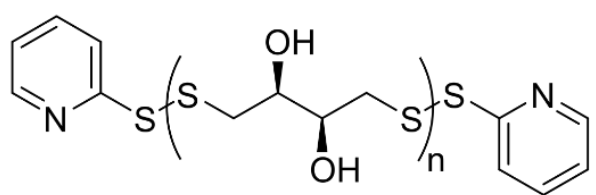

pDTT pyridinedisulfide end-cap

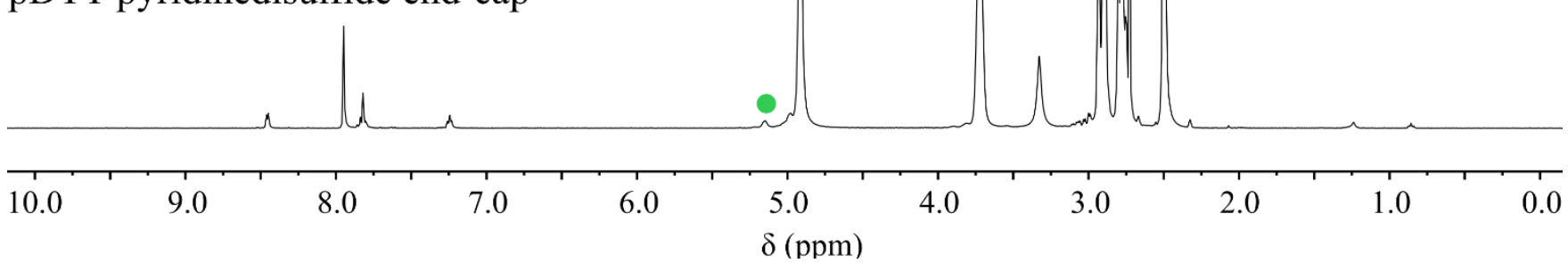

Figure S7. ${ }^{1} \mathrm{H}$-NMR spectra of $\mathrm{pDTT}_{20}$ before (bottom, black) and after (top, green) treatment with 2-mercaptoethanol (2 equiv.). Note that the $\mathrm{OH}$ peak $(\bullet)$ closest to the pyridine moiety disappears while the quartet ascribed to $-\mathrm{CH}_{2}-\mathrm{OH}(\bullet)$ in the end-cap of 2-mercaptoethanol appears at $3.64 \mathrm{ppm}$ upon replacement. In addition, the pyridine-2-thiol expelled is detected at $7.58,7.38$ and $6.77 \mathrm{ppm}$ (see also Figure S5). Residues of DMF released from $\mathrm{pDTT}_{20}$ are apparent from the beginning $(\delta=$ $7.92,2.92$ and $2.75 \mathrm{ppm})$, in addition to peaks from DMSO- $d_{6}(2.50 \mathrm{ppm})$ and water $(3.33 \mathrm{ppm})$. 
Supporting Information - Synthesis and Closed-Loop Recycling of Self-Immolative Poly(Dithiothreitol)

Spectra of undissolved pDTT 20 in $\mathrm{D}_{2} \mathrm{O}$ after 20 days $\left({ }^{1} \mathrm{H}-\mathrm{NMR}\right)$

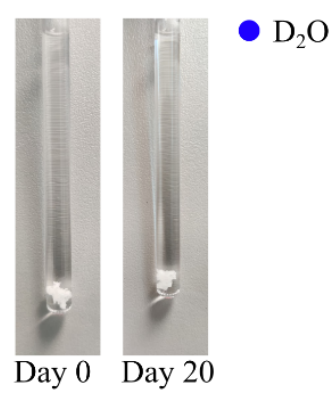

\begin{tabular}{|c|c|c|c|c|c|c|c|c|c|c|}
\hline 10.0 & 9.0 & 8.0 & 7.0 & 6.0 & $\begin{array}{c}5.0 \\
\delta(\mathrm{p})\end{array}$ & 4.0 & 3.0 & 2.0 & 1.0 & 0.0 \\
\hline
\end{tabular}

Figure S8. ${ }^{1} \mathrm{H}-\mathrm{NMR}$ spectra of $\mathrm{pDTT}_{20}$ in $\mathrm{D}_{2} \mathrm{O}$ after 20 days in solution. Pictures of the solid polymer in the water the day of submerging and after 20 days in solution (inset). 
Solid-state depolymerization of pDTT 20 submerged in $\mathrm{D}_{2} \mathrm{O}$ under inert argon atmosphere $\left({ }^{1} \mathrm{H}-\mathrm{NMR}\right)$

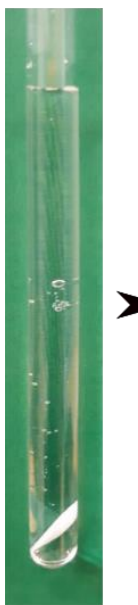

Day 0

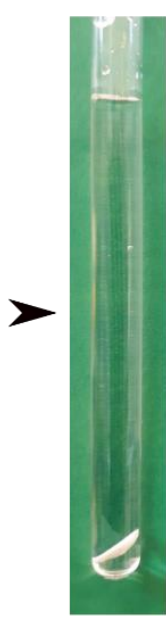

Day 1

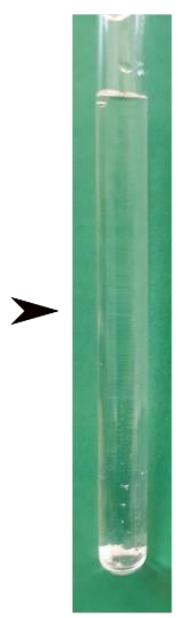

Day 2

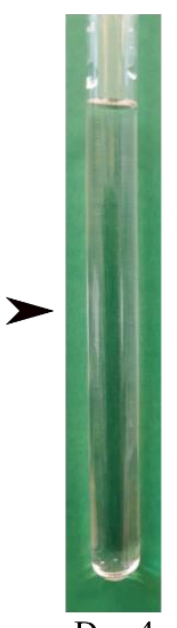

Day 4

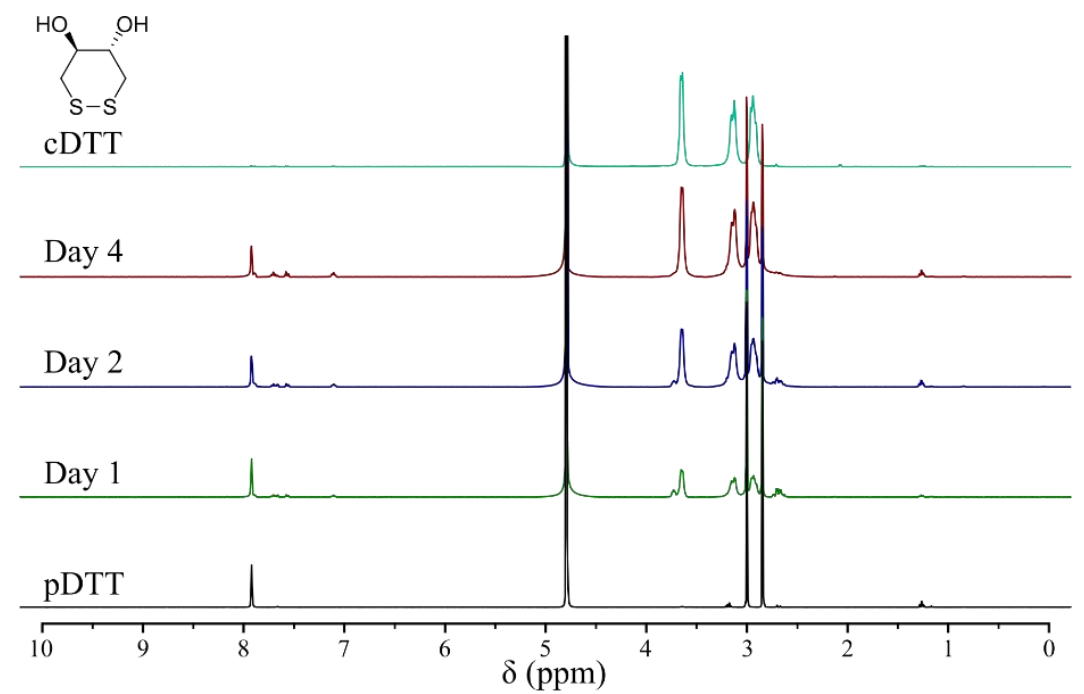

Figure S9. Photos showing a solid piece of $\mathrm{pDTT}_{20}$ submerged in $\mathrm{D}_{2} \mathrm{O}$ containing 1 equiv. $\mathrm{Et}_{3} \mathrm{~N}$ in an NMR tube before (day 0 ) and after addition of 2 equiv. DTT (days 1, 2 and 4). Corresponding ${ }^{1} \mathrm{H}-\mathrm{NMR}$ spectra of $\mathrm{pDTT}_{20}$ (bottom, black) along with a reference spectrum of cDTT in $\mathrm{D}_{2} \mathrm{O}$ (top, turquoise), noting that the -OH of cDTT, usually appearing at $\delta=5.22 \mathrm{ppm}$, is absent because of proton/deuterium exchange with the solvent. Residues of DMF released from pDTT $_{20}$ are apparent from the beginning $(\delta=7.92,2.92$ and $2.75 \mathrm{ppm})$ but exert no effect on the kinetics. In addition peaks are observed from DMSO- $d_{6}$ $(2.50 \mathrm{ppm})$ and water $(3.33 \mathrm{ppm})$. 
Depolymerization of $p D T T_{20}$ in $D M F(S E C)$

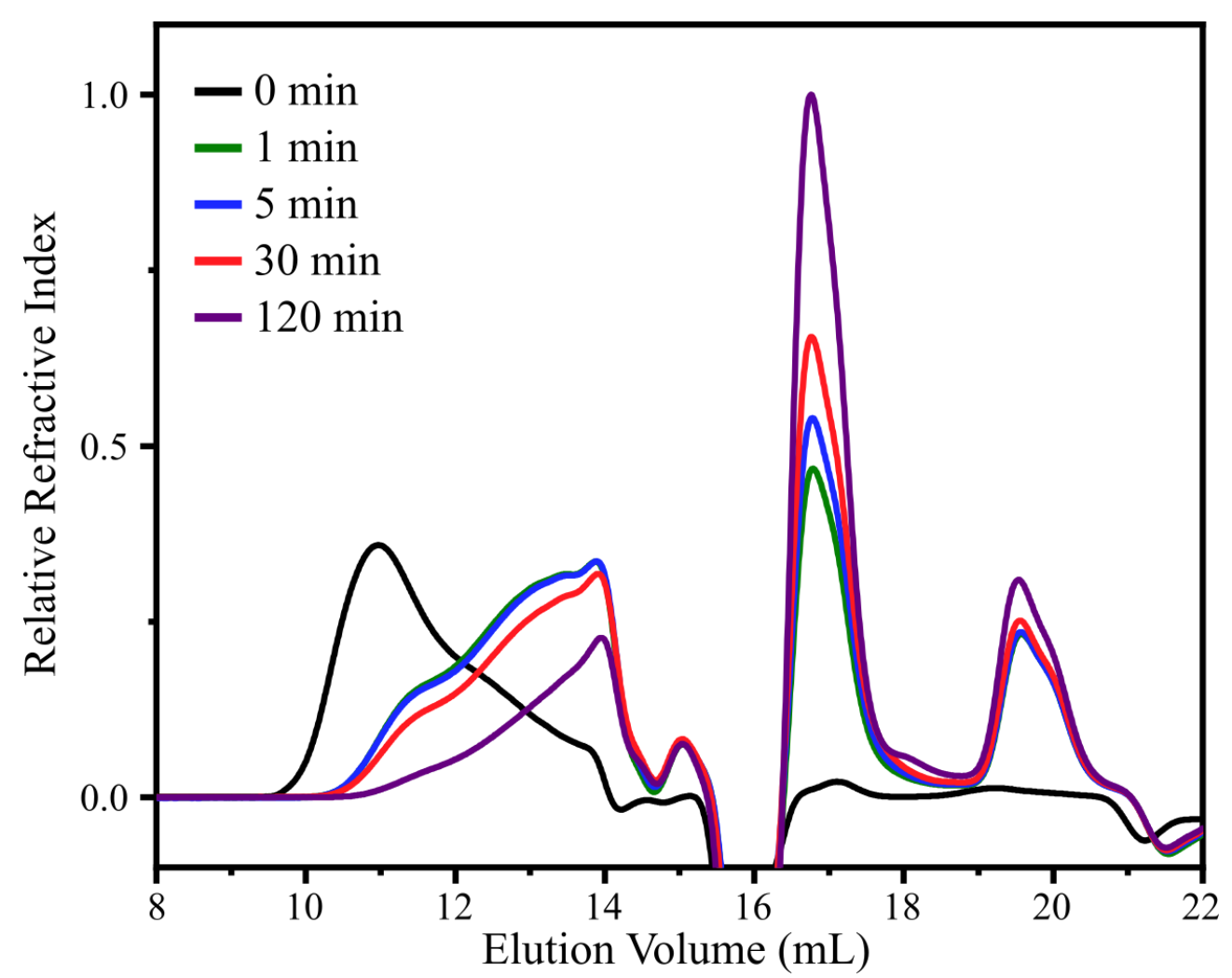

Figure S10. SEC traces of pDTT 20 in DMF after addition of 2 equiv. DTT under $\mathrm{N}_{2}$ atmosphere and quenched after $0,1,5$, 30 and 120 min using 4 equiv. DTDP. 
Supporting Information - Synthesis and Closed-Loop Recycling of Self-Immolative Poly(Dithiothreitol)

Spectra of DTDP, DTT and $c D T T\left({ }^{1} H-N M R\right)$

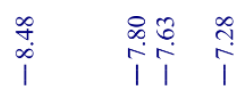

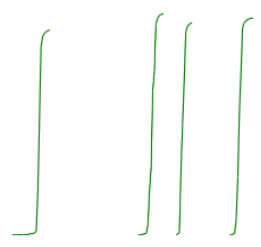<smiles>c1ccc(SSc2ccccn2)nc1</smiles>

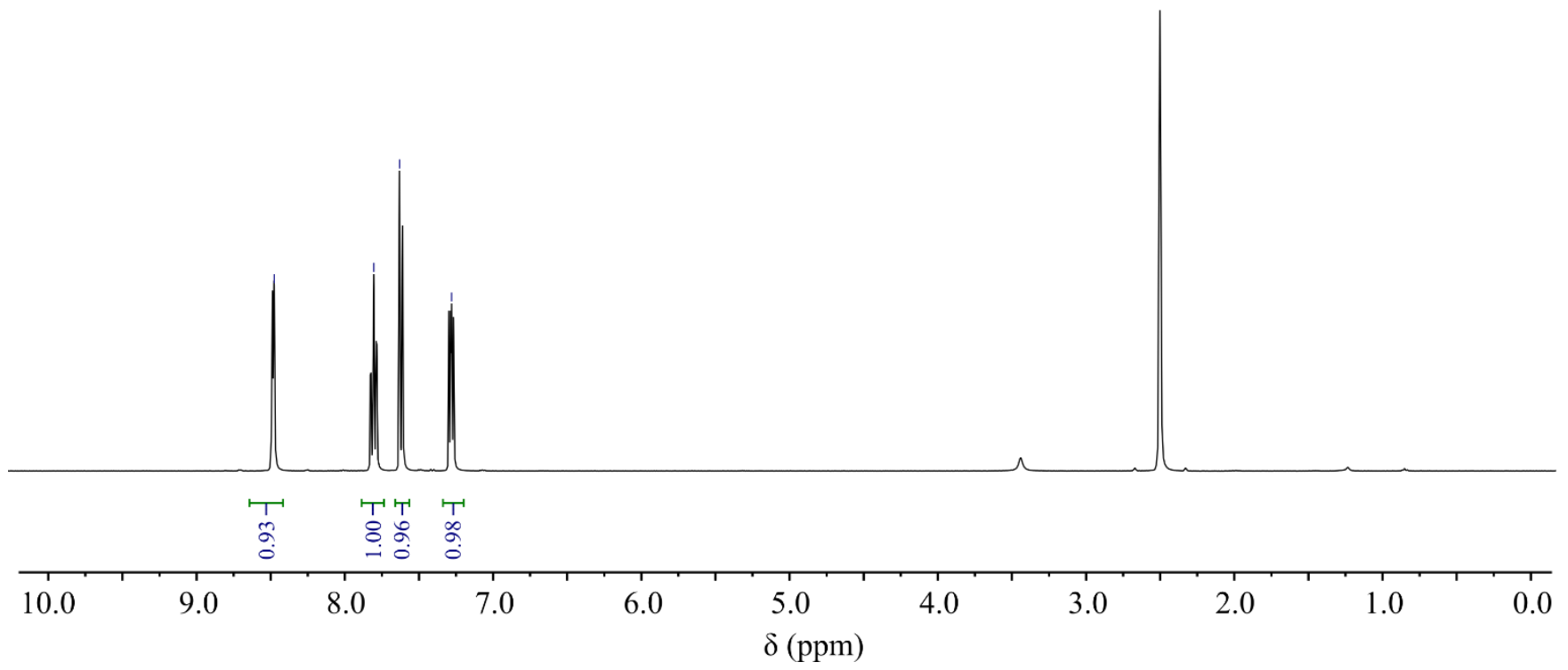

Figure S11. ${ }^{1} \mathrm{H}-\mathrm{NMR}$ spectrum of DTDP in DMSO- $d_{6}$. 
Supporting Information - Synthesis and Closed-Loop Recycling of Self-Immolative Poly(Dithiothreitol)

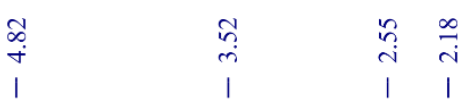<smiles>O[C@H](CS)[C@@H](O)CS</smiles>
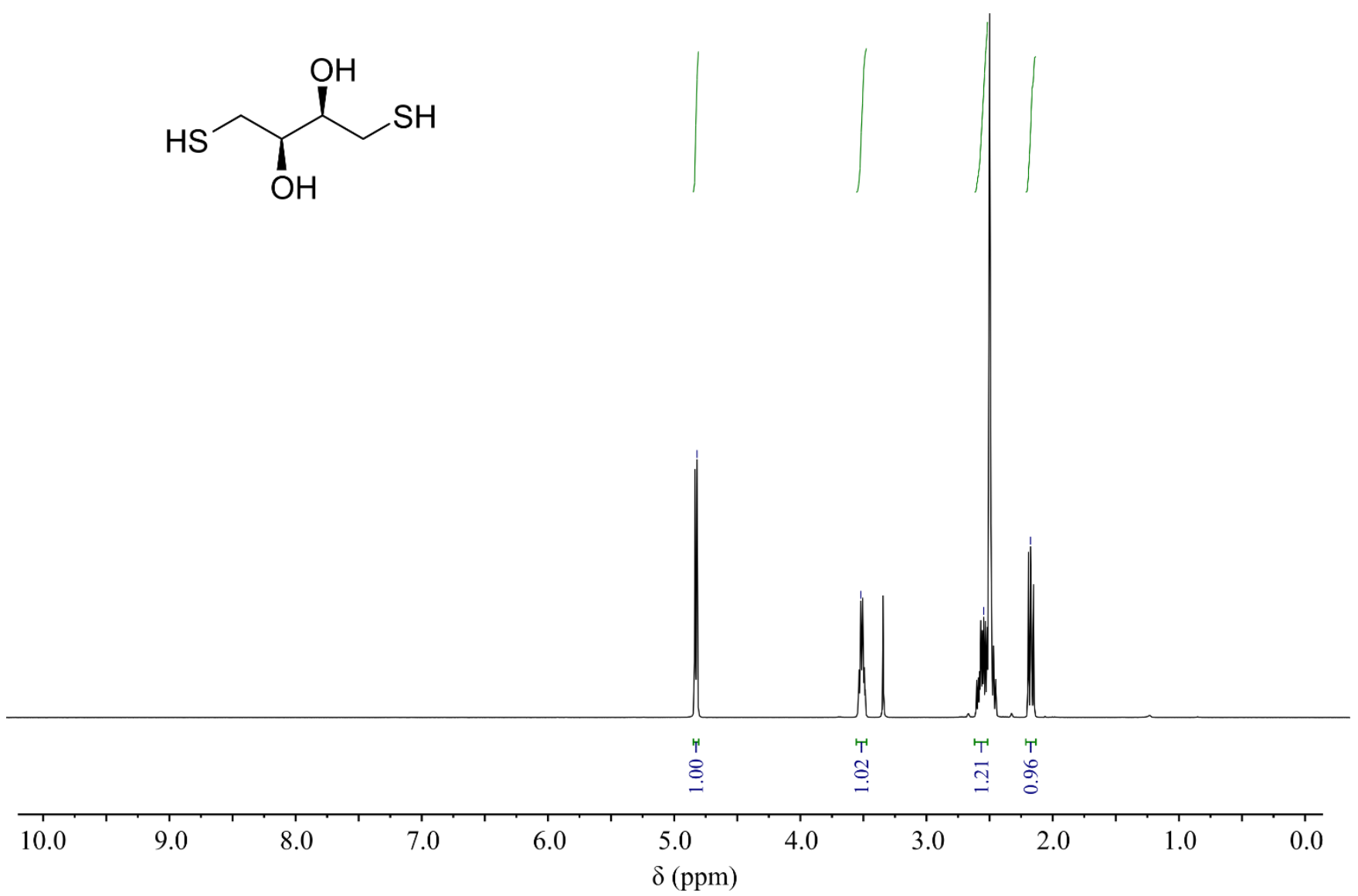

Figure S12. ${ }^{1} \mathrm{H}-\mathrm{NMR}$ spectrum of DTT in DMSO- $d_{6}$. 
Supporting Information - Synthesis and Closed-Loop Recycling of Self-Immolative Poly(Dithiothreitol)
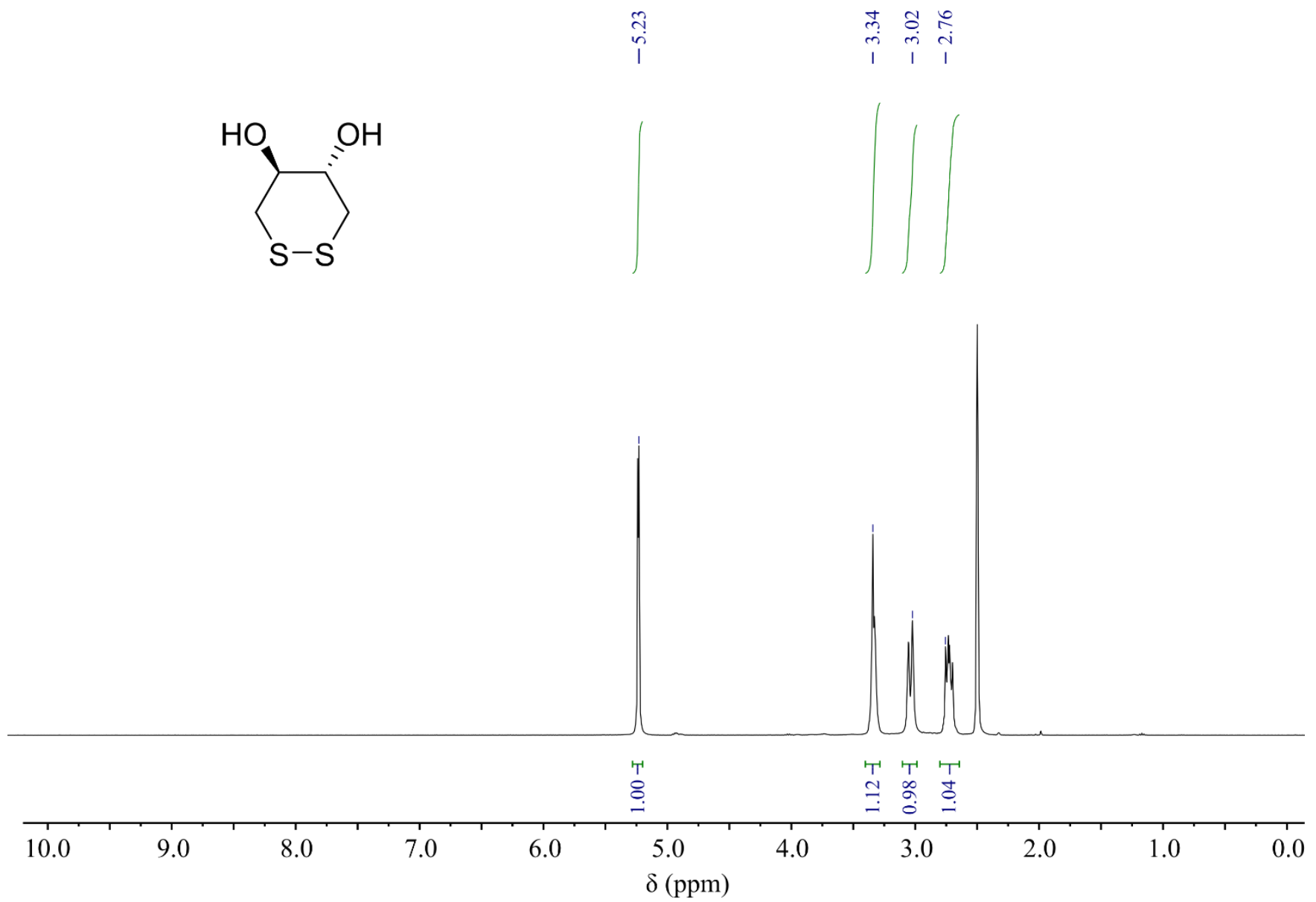

Figure S13. ${ }^{1} \mathrm{H}-\mathrm{NMR}$ spectrum of cDTT in DMSO- $d_{6}$. 
Supporting Information - Synthesis and Closed-Loop Recycling of Self-Immolative Poly(Dithiothreitol)

Depolymerization of pDTT 20 under ambient conditions in DMSO $\left({ }^{1} \mathrm{H}-\mathrm{NMR}\right)$

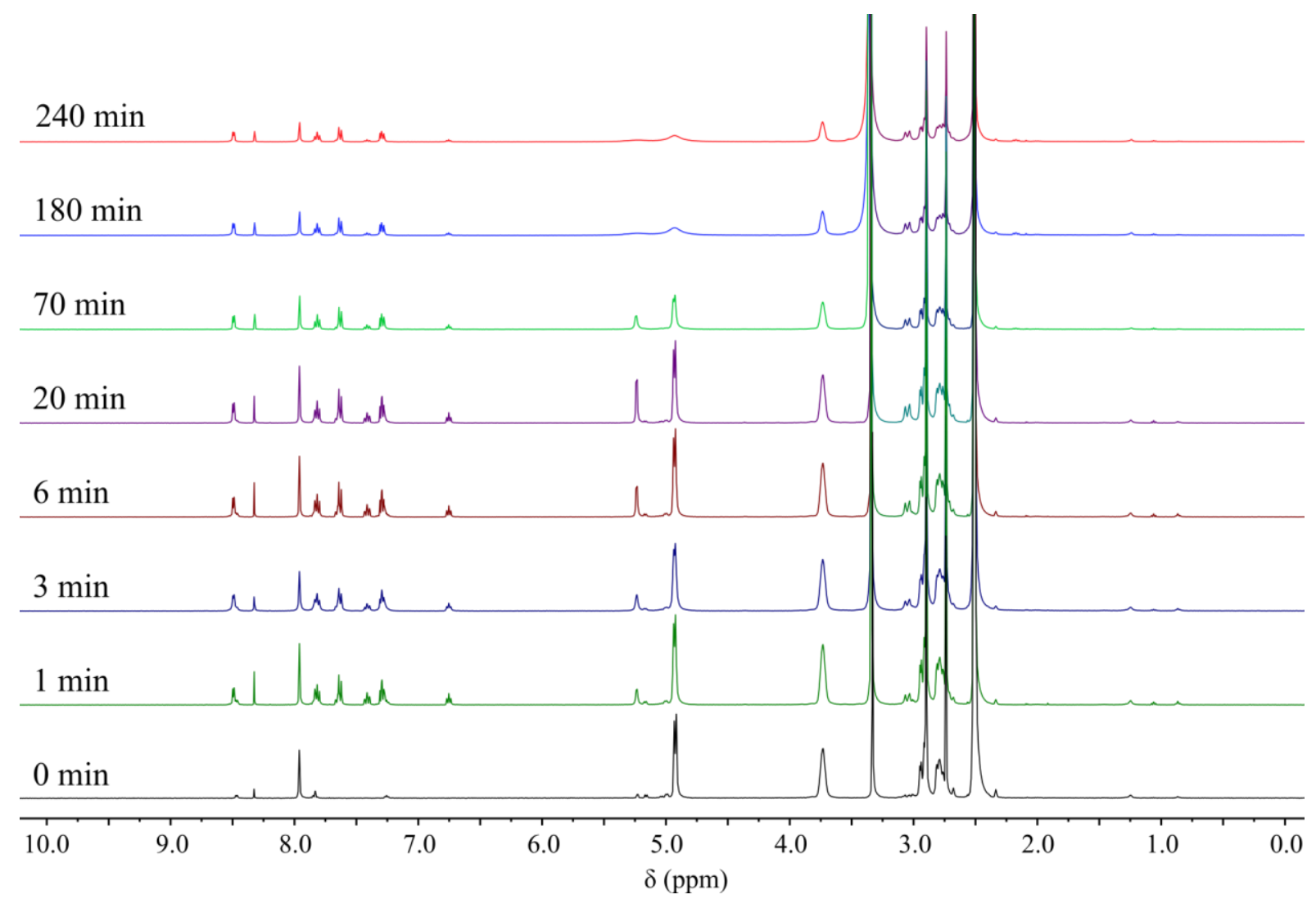

Figure S14. ${ }^{1} \mathrm{H}-\mathrm{NMR}$ spectra of pDTT 20 to monitor its depolymerization under ambient conditions (i.e. oxygen is present) in DMSO- $d_{6}$ after addition of 2 equiv. DTT. Aliquots were transferred after 1, 3, 6, 20, 70, 180 and 240 min from the polymer solution to a solution with DTDP in excess to quench the depolymerization. Residues of DMF released from pDTT 20 are apparent from the beginning $(\delta=7.92,2.92$ and $2.75 \mathrm{ppm})$, in addition to peaks from DMSO- $d_{6}(2.50 \mathrm{ppm}), \mathrm{CHCl}_{3}(8.32$ $\mathrm{ppm})$ and water (3.33 ppm). 
Supporting Information - Synthesis and Closed-Loop Recycling of Self-Immolative Poly(Dithiothreitol)

Depolymerization of pDTT 20 under inert conditions in DMSO $\left({ }^{1} \mathrm{H}-\mathrm{NMR}\right)$

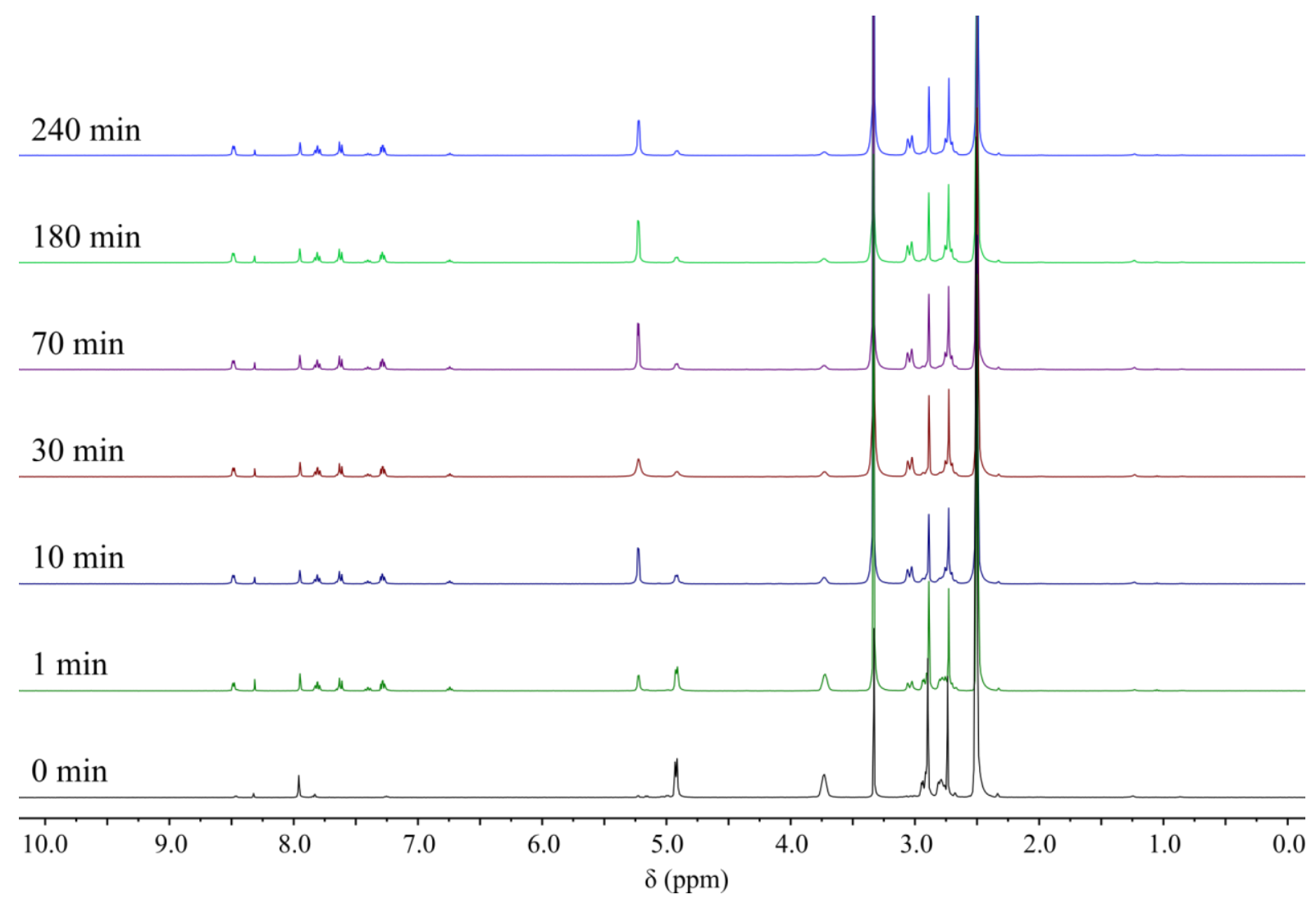

Figure S15. ${ }^{1} \mathrm{H}-\mathrm{NMR}$ spectra of $\mathrm{pDTT}_{20}$ to monitor its depolymerization under ambient conditions in $\mathrm{N}_{2}$-purged DMSO- $d_{6}$ after addition of 2 equiv. DTT. Aliquots were transferred after 1, 10, 30, 70, 180 and $240 \mathrm{~min}$ from the polymer solution to a solution with DTDP in excess to quench the depolymerization. Residues of DMF released from $\mathrm{pDTT}_{20}$ are apparent from the beginning $(\delta=7.92,2.92$ and $2.75 \mathrm{ppm})$, in addition to peaks from DMSO- $d_{6}(2.50 \mathrm{ppm}), \mathrm{CHCl}_{3}(8.32 \mathrm{ppm})$ and water (3.33 ppm). 
Supporting Information - Synthesis and Closed-Loop Recycling of Self-Immolative Poly(Dithiothreitol)

Depolymerization of pDTT 20 under ambient conditions base catalyzed in DMSO $\left({ }^{1} \mathrm{H}-\mathrm{NMR}\right)$

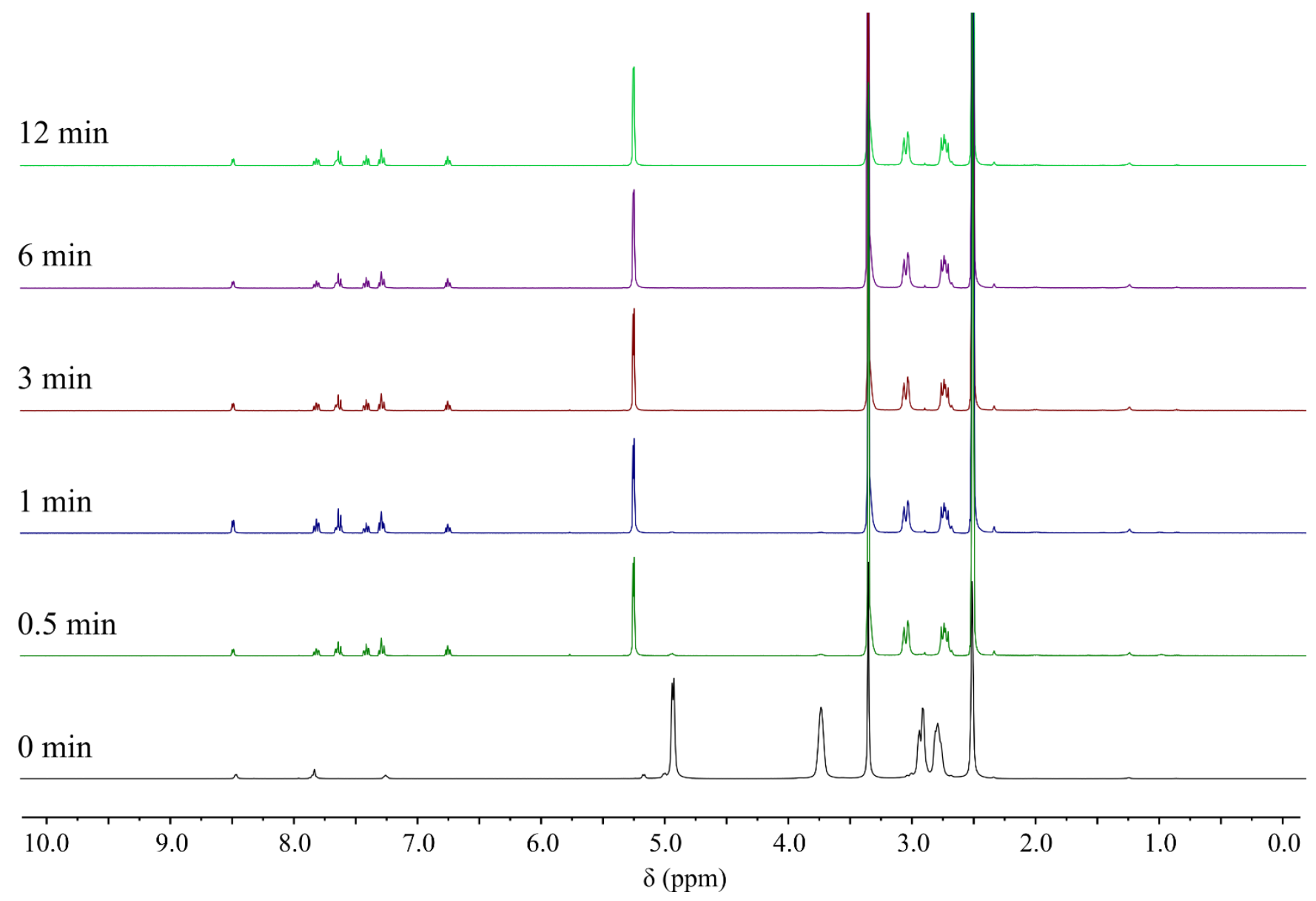

Figure S16. ${ }^{1} \mathrm{H}-\mathrm{NMR}$ spectra of $\mathrm{pDTT}_{20}$ to monitor its depolymerization under ambient conditions (i.e. oxygen is present) in DMSO- $d_{6}$ after addition of 2 equiv. DTT and 1 equiv. Et $3 \mathrm{~N}$. Aliquots were transferred after $0.5,1,3,6$, and 12 min from the polymer solution to a solution with DTDP in excess to quench the depolymerization. Residues of DMF released from pDTT 20 are apparent from the beginning $(\delta=7.92,2.92$ and $2.75 \mathrm{ppm})$, in addition to peaks from DMSO- $d_{6}(2.50 \mathrm{ppm}), \mathrm{CHCl}_{3}(8.32$ $\mathrm{ppm})$ and water (3.33 ppm). 
Supporting Information - Synthesis and Closed-Loop Recycling of Self-Immolative Poly(Dithiothreitol)

Degradation of pDTT 20 in neutral, basic, acidic and heated medium in DMSO $\left({ }^{1} \mathrm{H}-\mathrm{NMR}\right)$

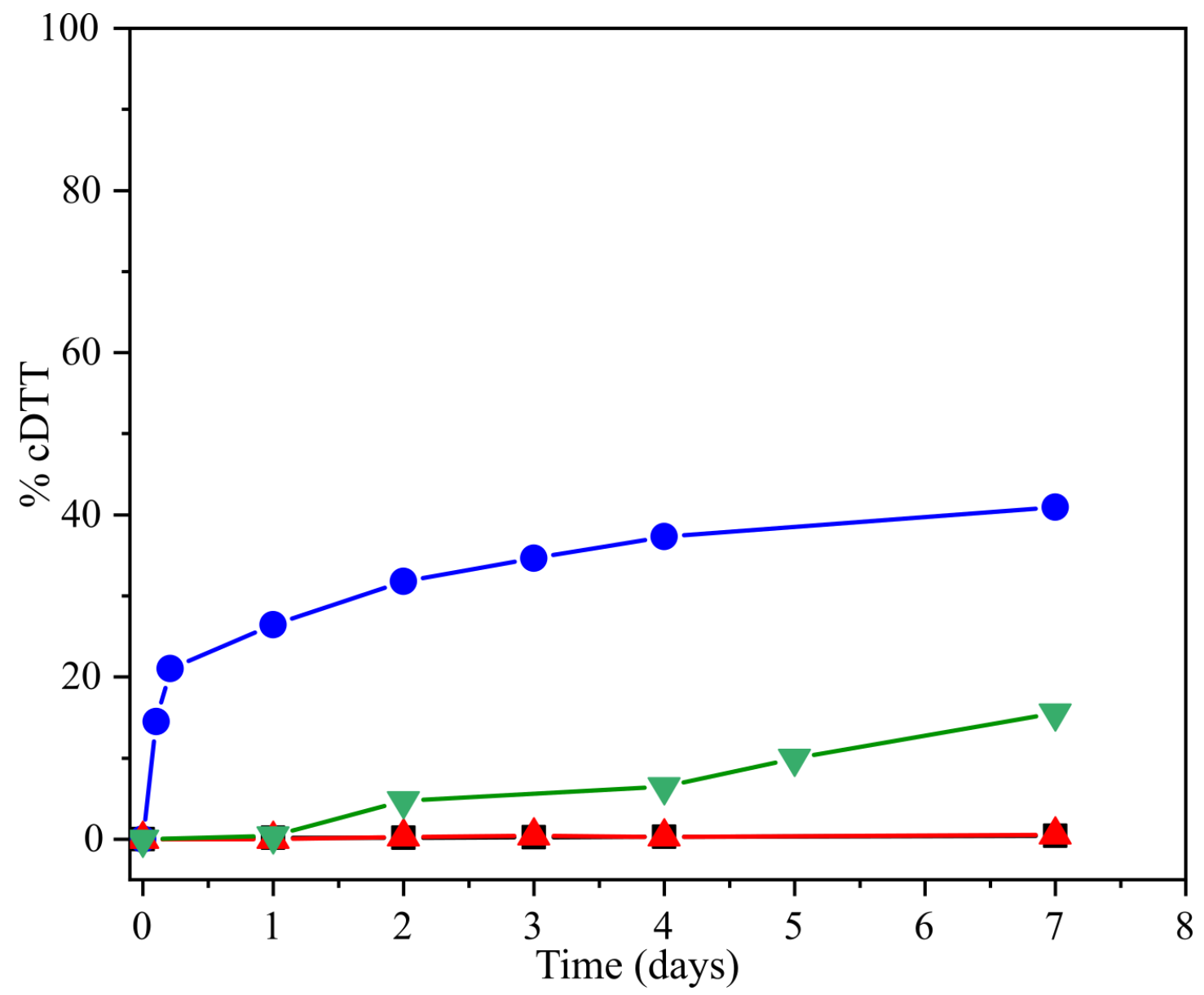

Figure S17. Kinetic traces showing the percentage of cDTT (determined by ${ }^{1} \mathrm{H}-\mathrm{NMR}$ ) formed from $\mathrm{pDTT} \mathrm{T}_{20}$ as a function of time in DMSO- $d_{6}$ under ambient conditions at room temperature $(\boldsymbol{\nabla})$ or $60{ }^{\circ} \mathrm{C}(\boldsymbol{\nabla})$ and in the presence of $2 \mathrm{mM} \mathrm{Et}_{3} \mathrm{~N}(\bullet)$ or $2 \mathrm{mM}$ acetic acid $(\boldsymbol{\Lambda})$. 


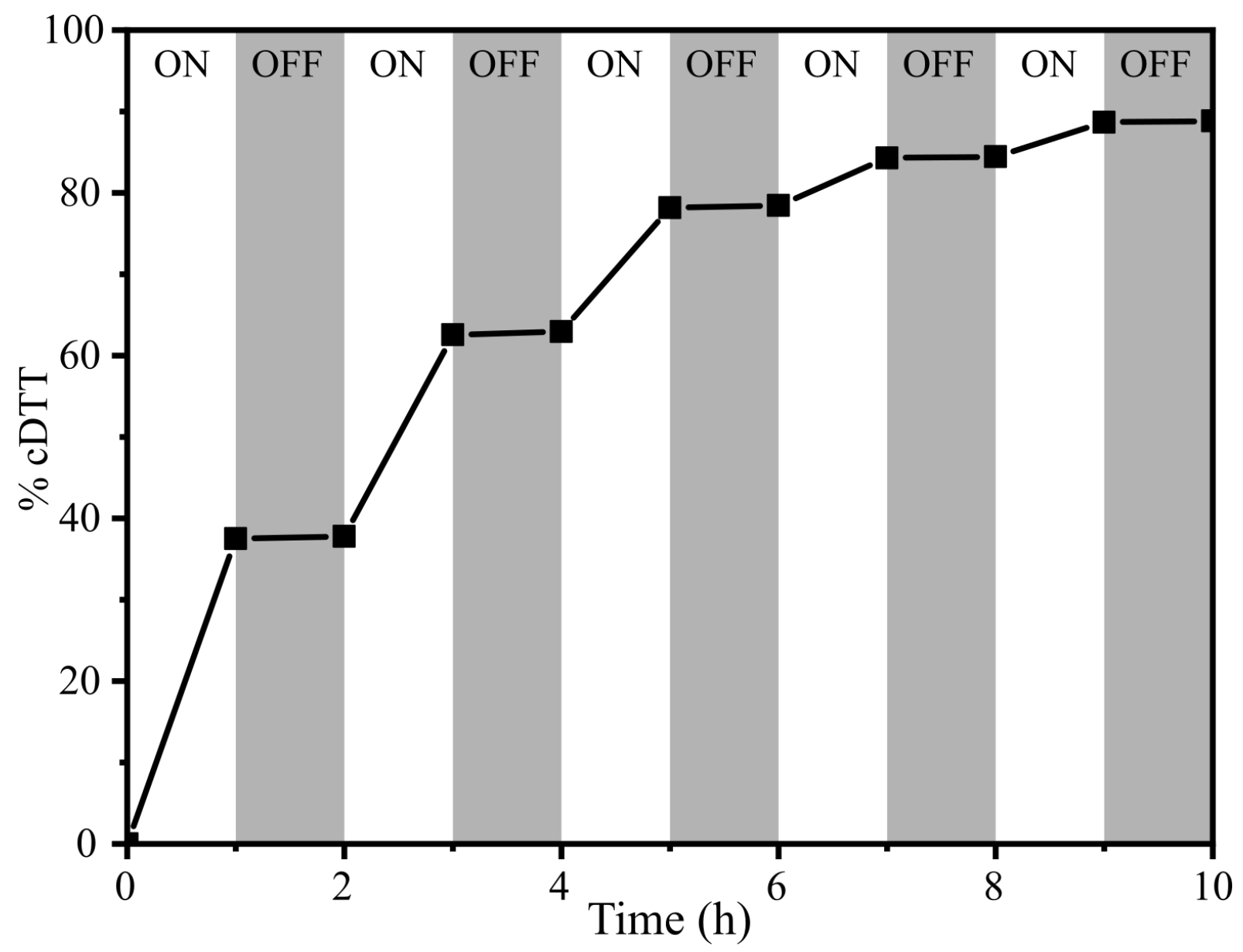

Figure S18. Monitoring of the percentage of cDTT (determined by ${ }^{1} \mathrm{H}-\mathrm{NMR}$ ) formed from the degradation of pDTT 20 as a result of UV irradiation ( $\lambda_{\max }=365 \mathrm{~nm}$; "ON") and without exposure to light ("OFF"). 


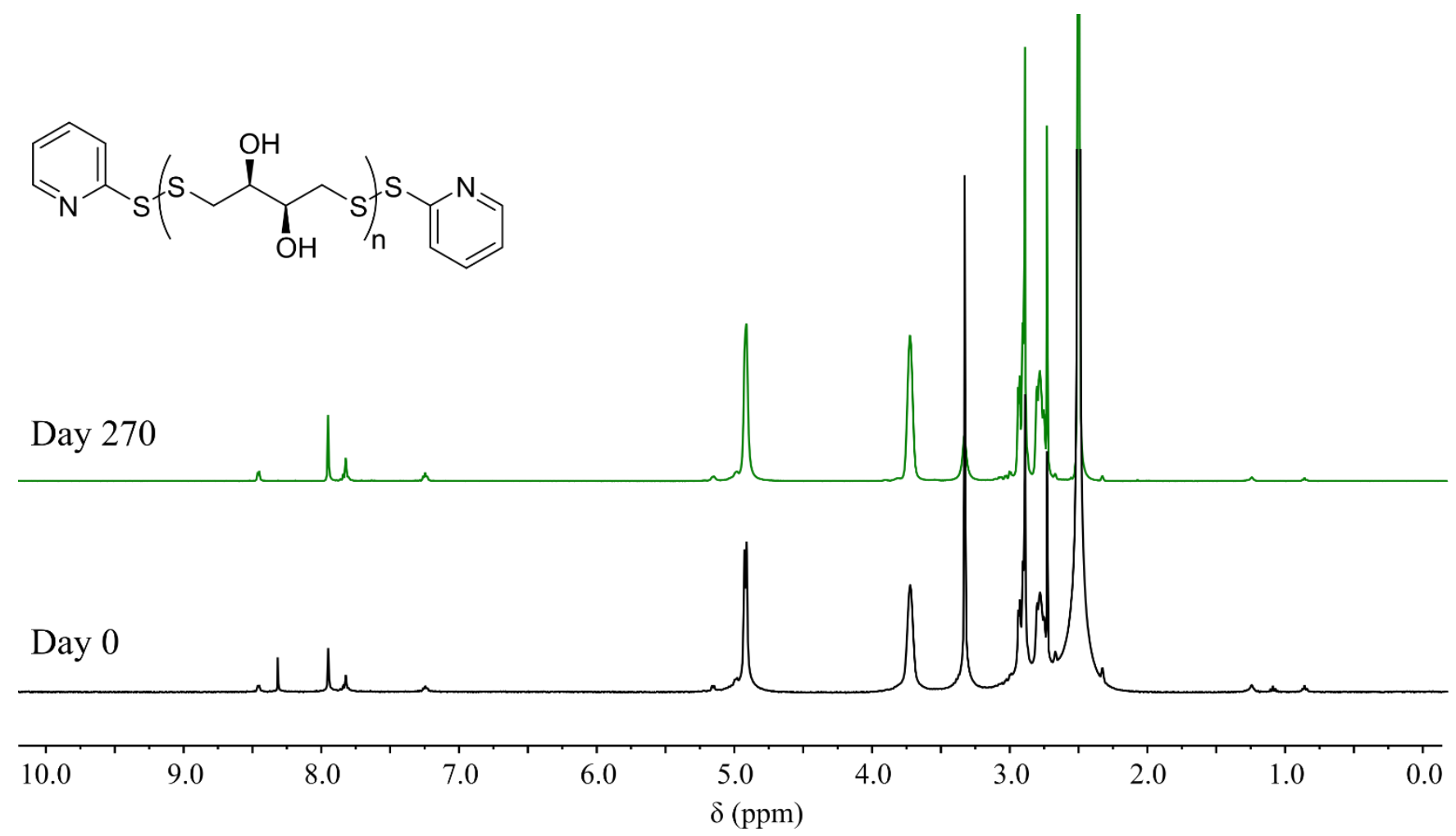

Figure S19. ${ }^{1} \mathrm{H}-\mathrm{NMR}$ spectra of $\mathrm{pDTT}_{20}$ recorded in DMSO- $d_{6}$ on the day of polymerization (bottom, black) and after storage in solid state for 270 days under ambient conditions (top, green). The only change seen to the spectrum is the disappearance of the $\mathrm{CHCl}_{3}$ impurity at $8.32 \mathrm{ppm}$ due to evaporation of solvent residues. Residues of DMF released from $\mathrm{pDTT}_{20}$ are apparent from the beginning $(\delta=7.92,2.92$ and $2.75 \mathrm{ppm})$, in addition to peaks from DMSO- $d_{6}(2.50 \mathrm{ppm}), \mathrm{CHCl}_{3}(8.32$ $\mathrm{ppm})$ and water (3.33 ppm). 
Supporting Information - Synthesis and Closed-Loop Recycling of Self-Immolative Poly(Dithiothreitol)

Spectrum of pyridine-2-thiol $\left({ }^{1} \mathrm{H}-\mathrm{NMR}\right)$

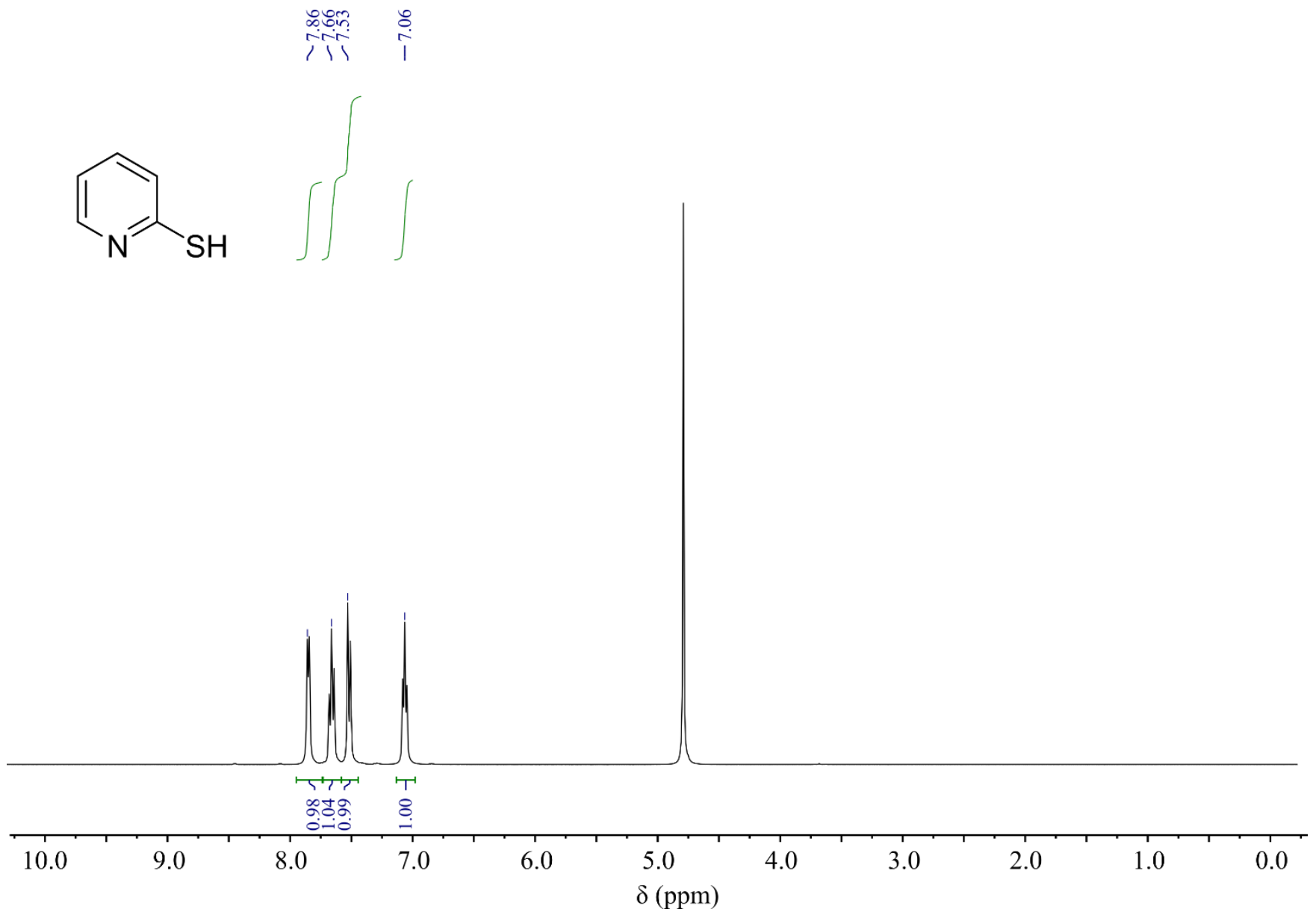

Figure S20. ${ }^{1} \mathrm{H}-\mathrm{NMR}$ spectrum of pyridine-2-thiol (reference compound) in $\mathrm{D}_{2} \mathrm{O}$. 
Supporting Information - Synthesis and Closed-Loop Recycling of Self-Immolative Poly(Dithiothreitol)

Spectrum of cDTT $\left({ }^{1} H-N M R\right)$

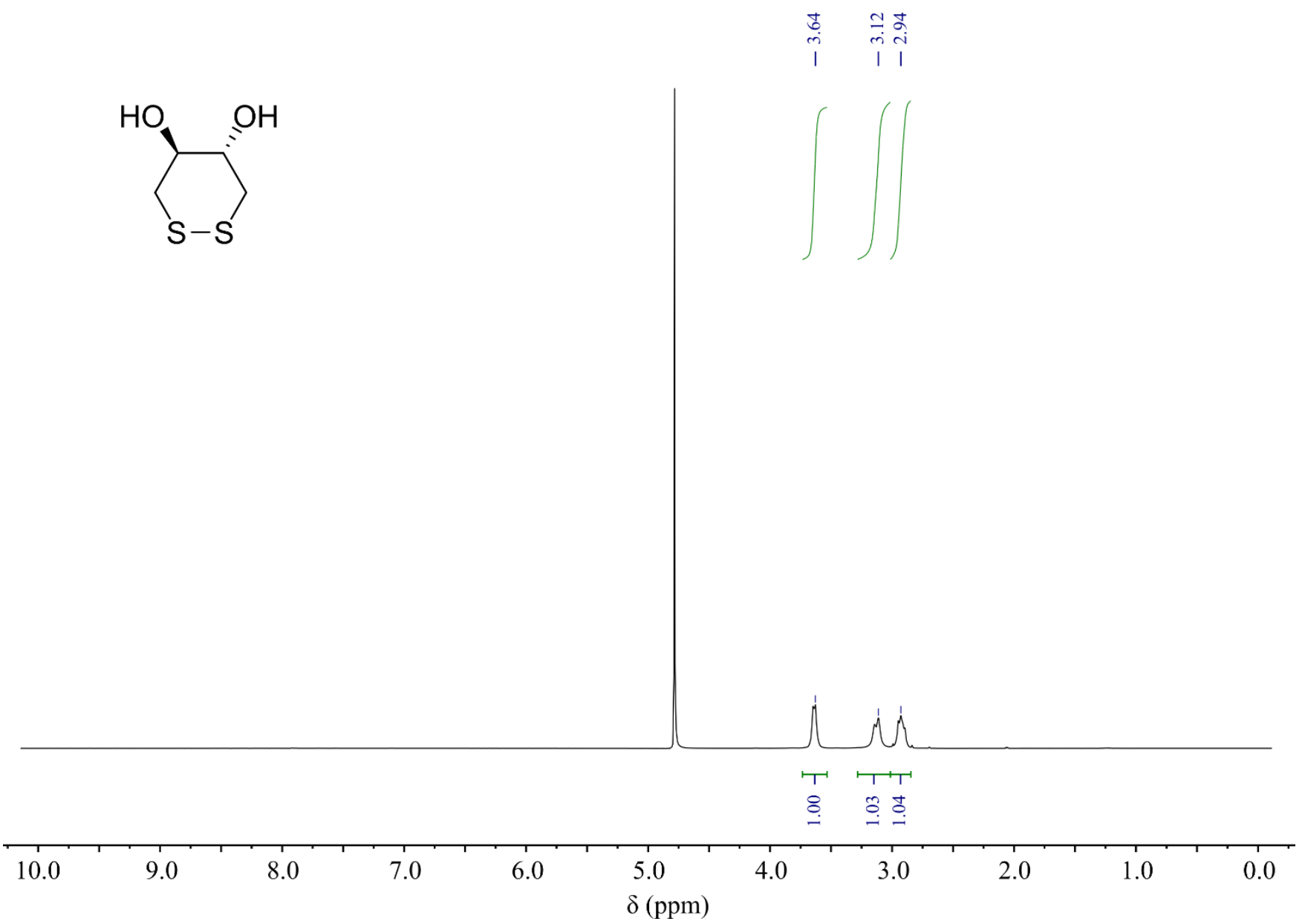

Figure S21. ${ }^{1} \mathrm{H}-\mathrm{NMR}$ spectrum of cDTT (reference compound) in $\mathrm{D}_{2} \mathrm{O}$. 
TGA and DTG analysis of pDTT 20

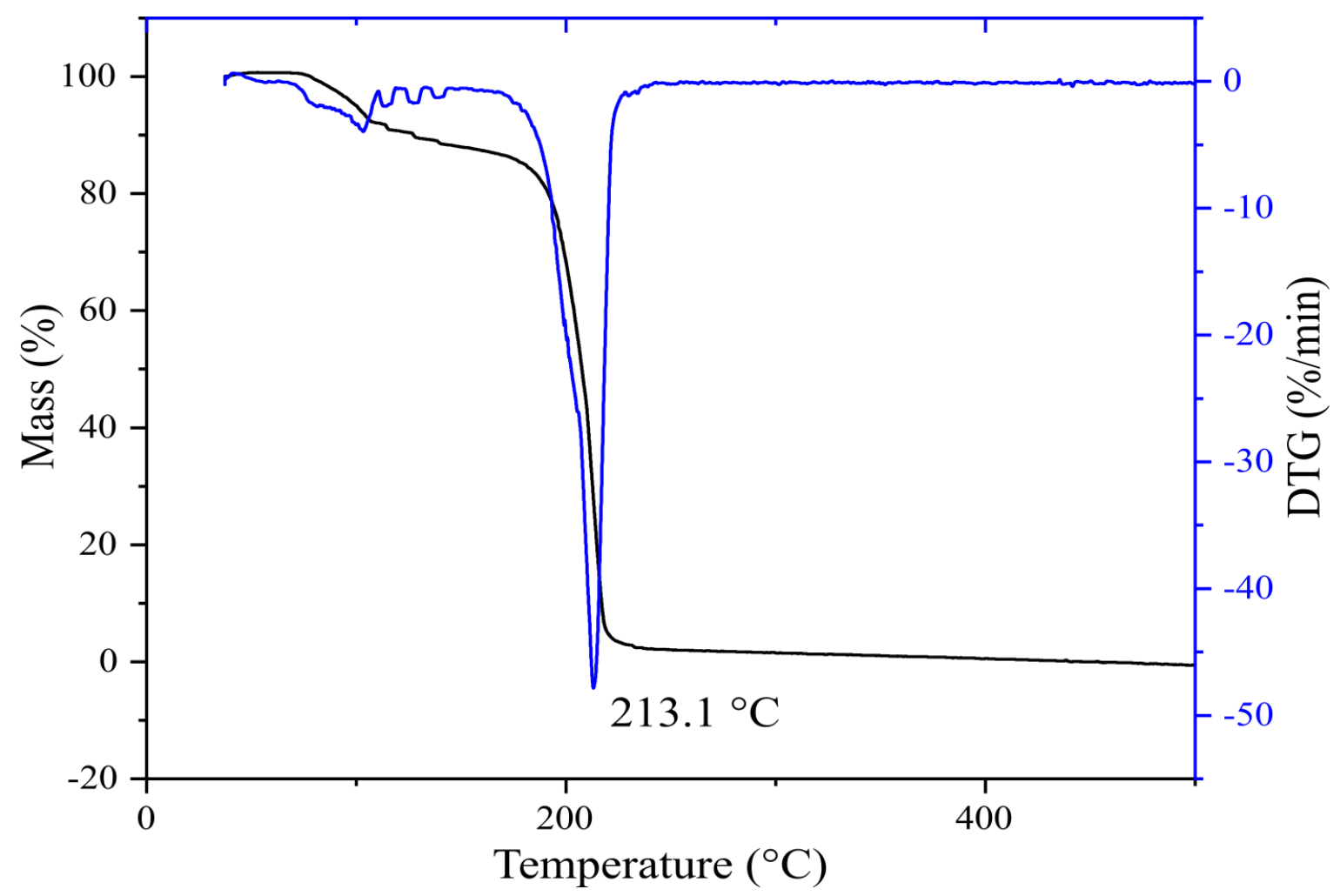

Figure S22. TGA of $\mathrm{pDTT}_{20}$ (precipitated from THF) where the mass loss occurring before $120{ }^{\circ} \mathrm{C}(\sim 10 \%)$ is attributed to the already identified impurities (see Figure S29) of THF (bp: $66^{\circ} \mathrm{C}$ ) and water (bp: $100{ }^{\circ} \mathrm{C}$ ). The rapid and extensive weight loss ( $90 \%)$ at $200-220^{\circ} \mathrm{C}$ signifies the thermal decomposition of $\mathrm{pDTT}_{20}$ where the maximum rate of decomposition occurs at $213{ }^{\circ} \mathrm{C}$ according to DTG analysis. 
MTDSC analysis of pDTT 20

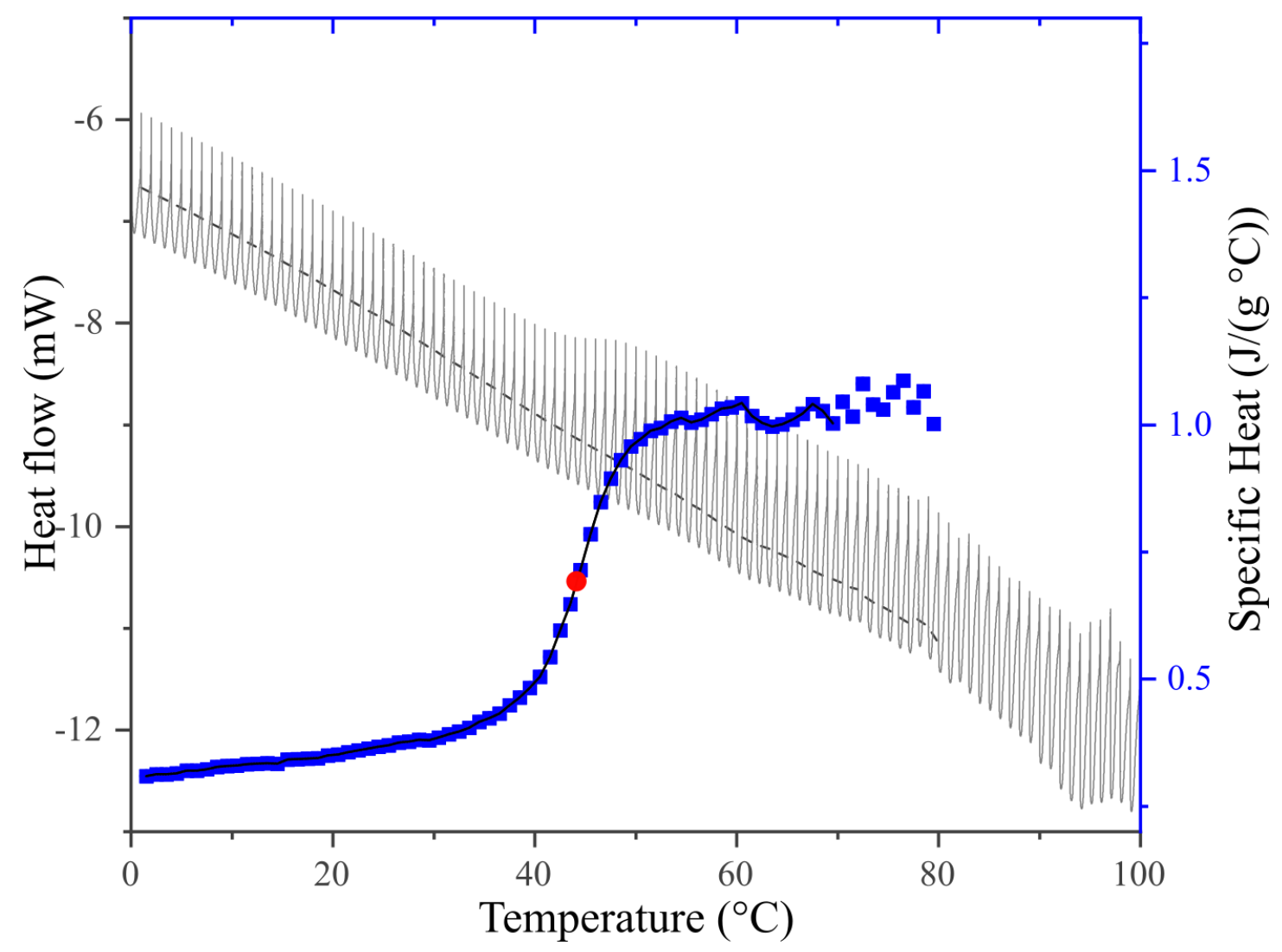

Figure S23. MTDSC analysis of pDTT 20 (precipitated from THF) exposed to a solvent stripping for 20 min at $100{ }^{\circ} \mathrm{C}$ prior to the actual experiment. Measured heat flow (black), isothermal heat flow base line (dashed black line), calculated specific heat $(\square)$ and $T_{\mathrm{g}}(\bullet)$. 
Chromatograms of pDTT $10-200$ (SEC)

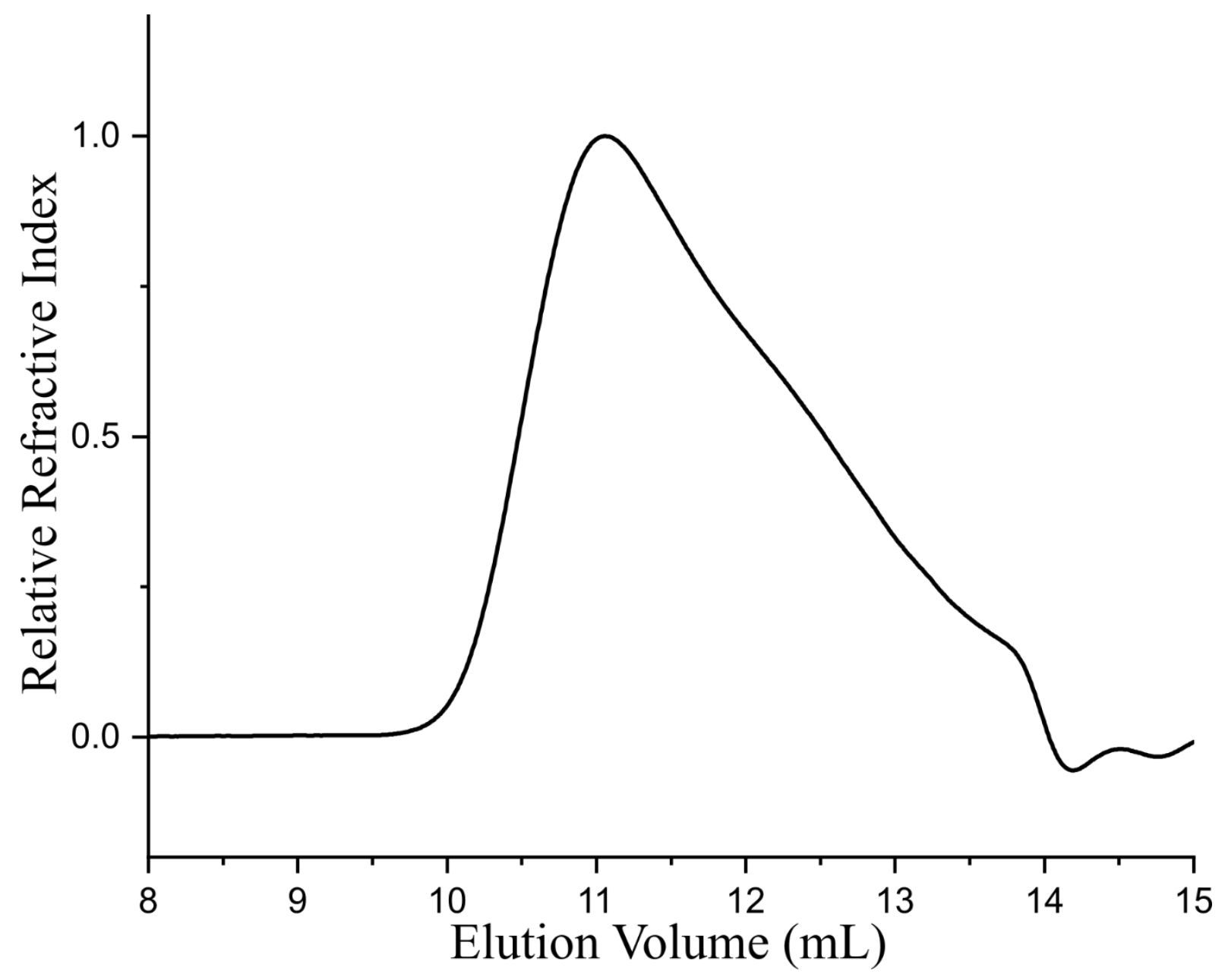

Figure S24. SEC trace of $\mathrm{pDTT}_{10}$ in DMF. 


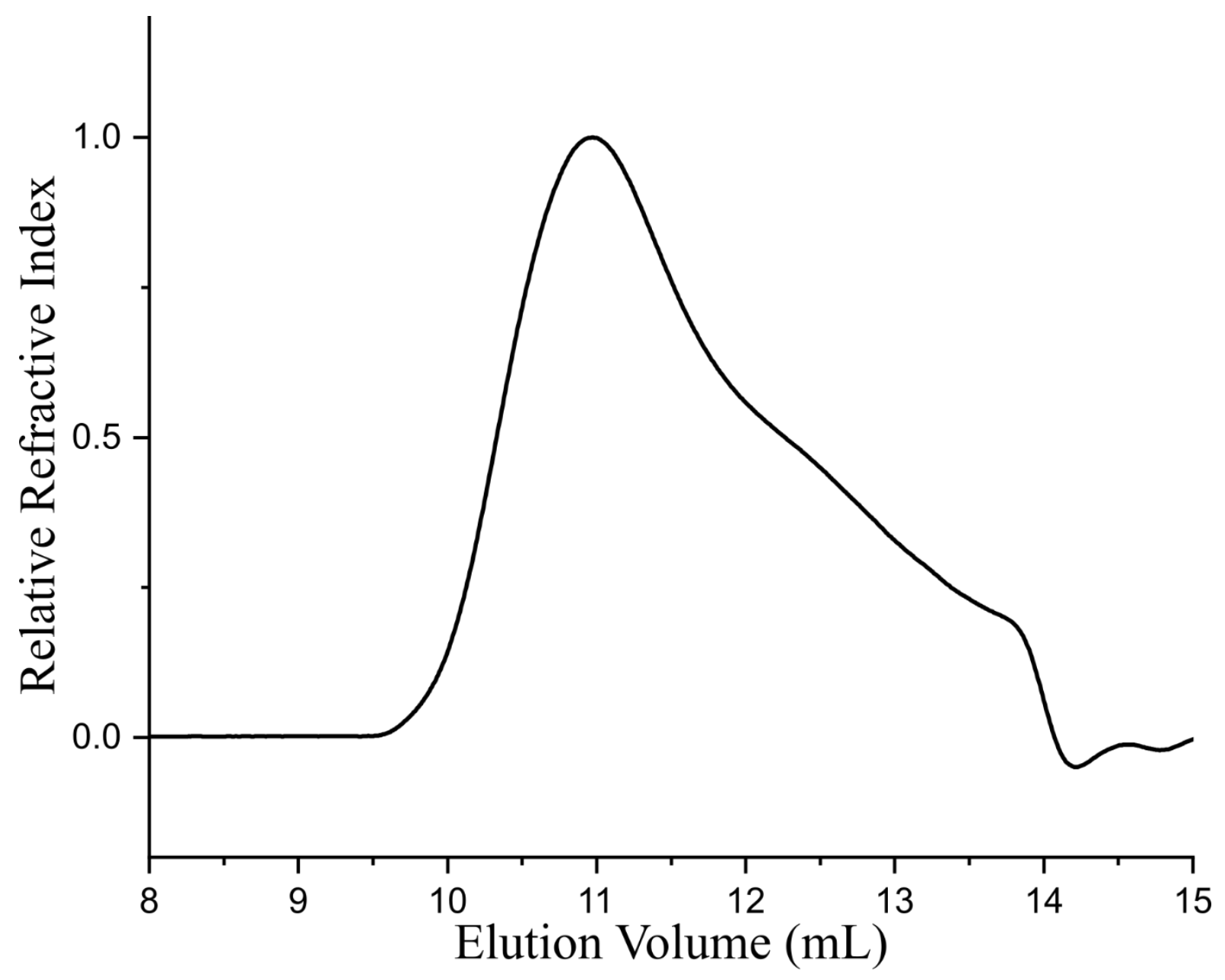

Figure S25. SEC trace of $\mathrm{pDTT}_{20}$ in DMF. 


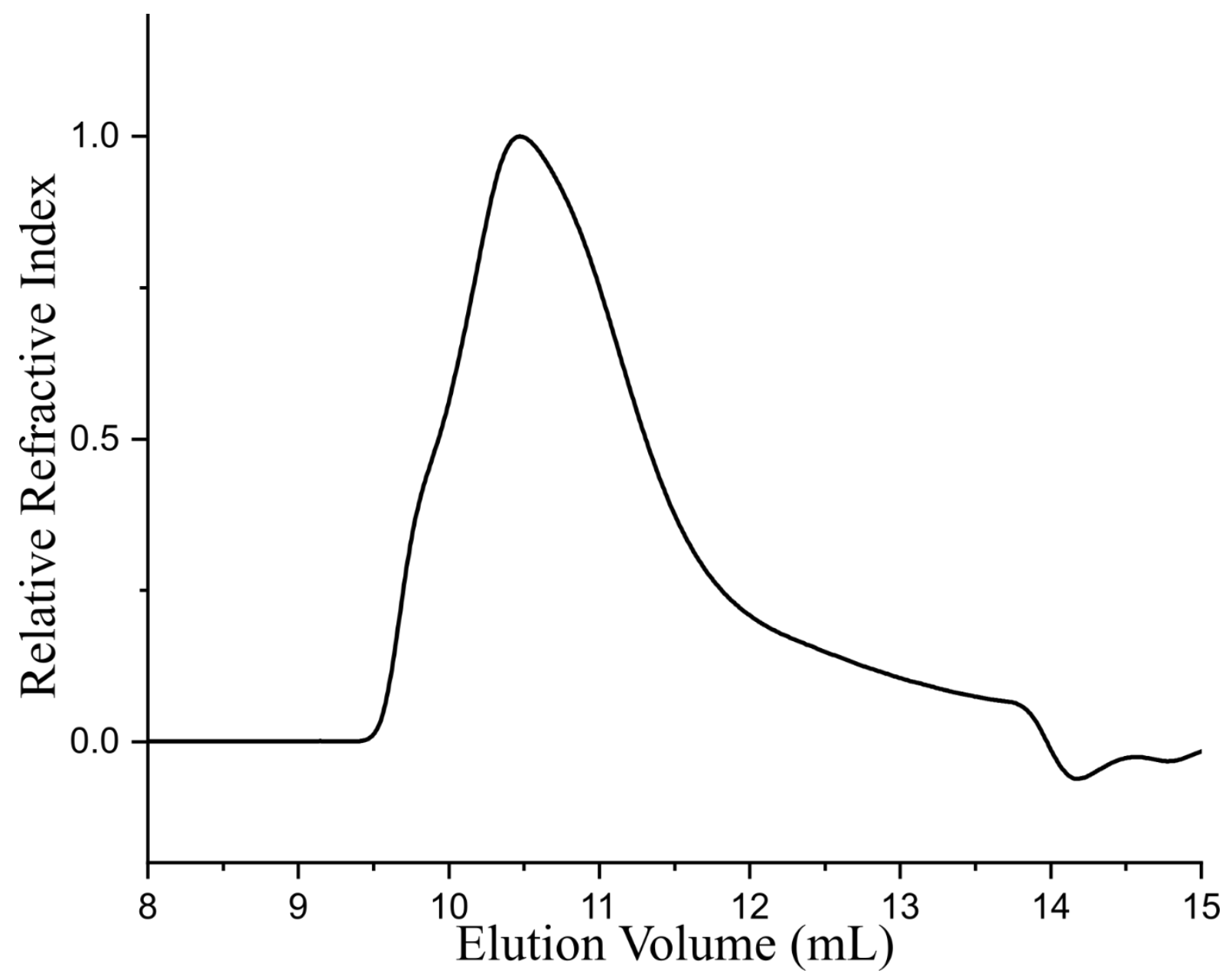

Figure S26. SEC trace of $\mathrm{pDTT}_{40}$ in DMF. 


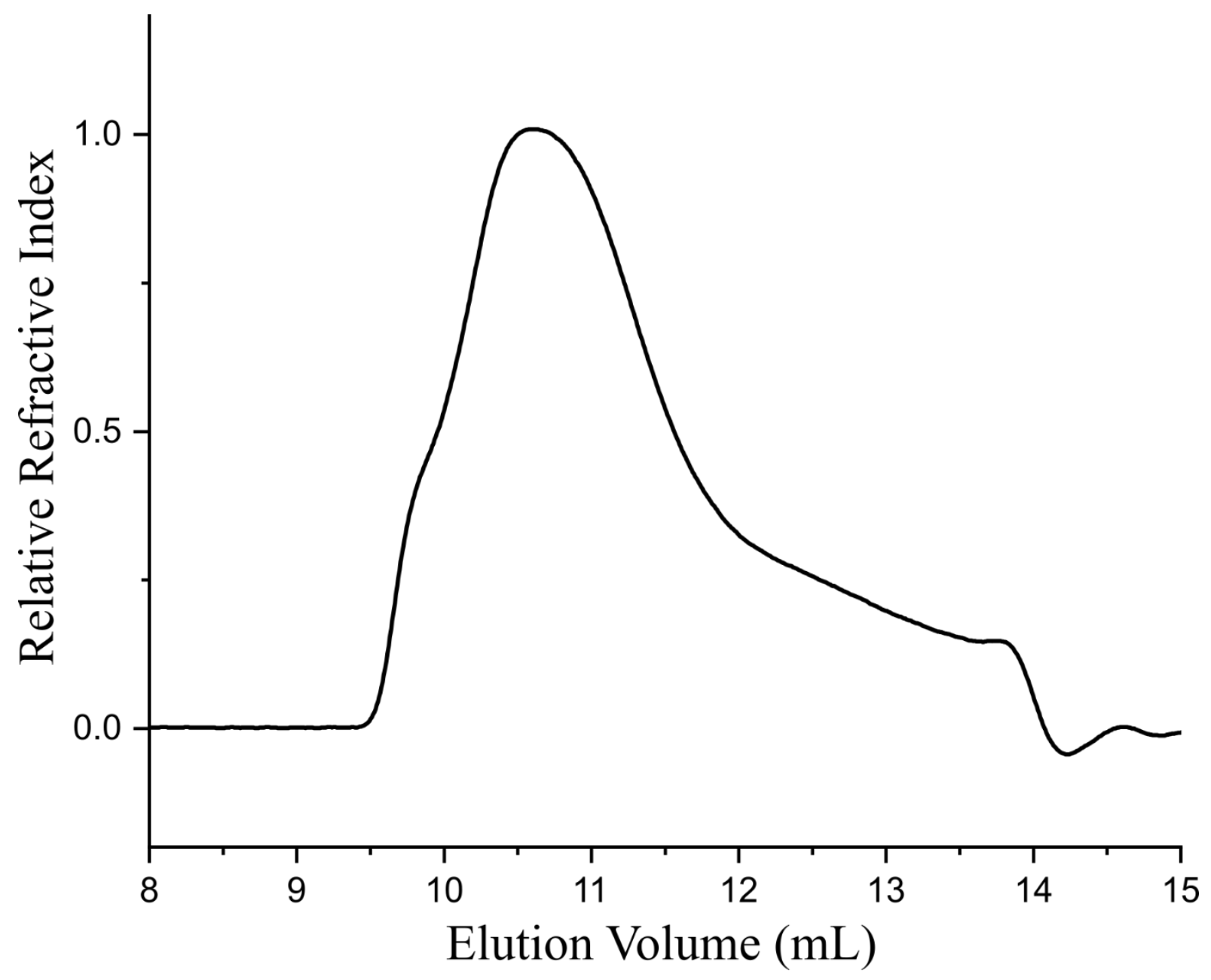

Figure S27. SEC trace of pDTT $_{100}$ in DMF. 


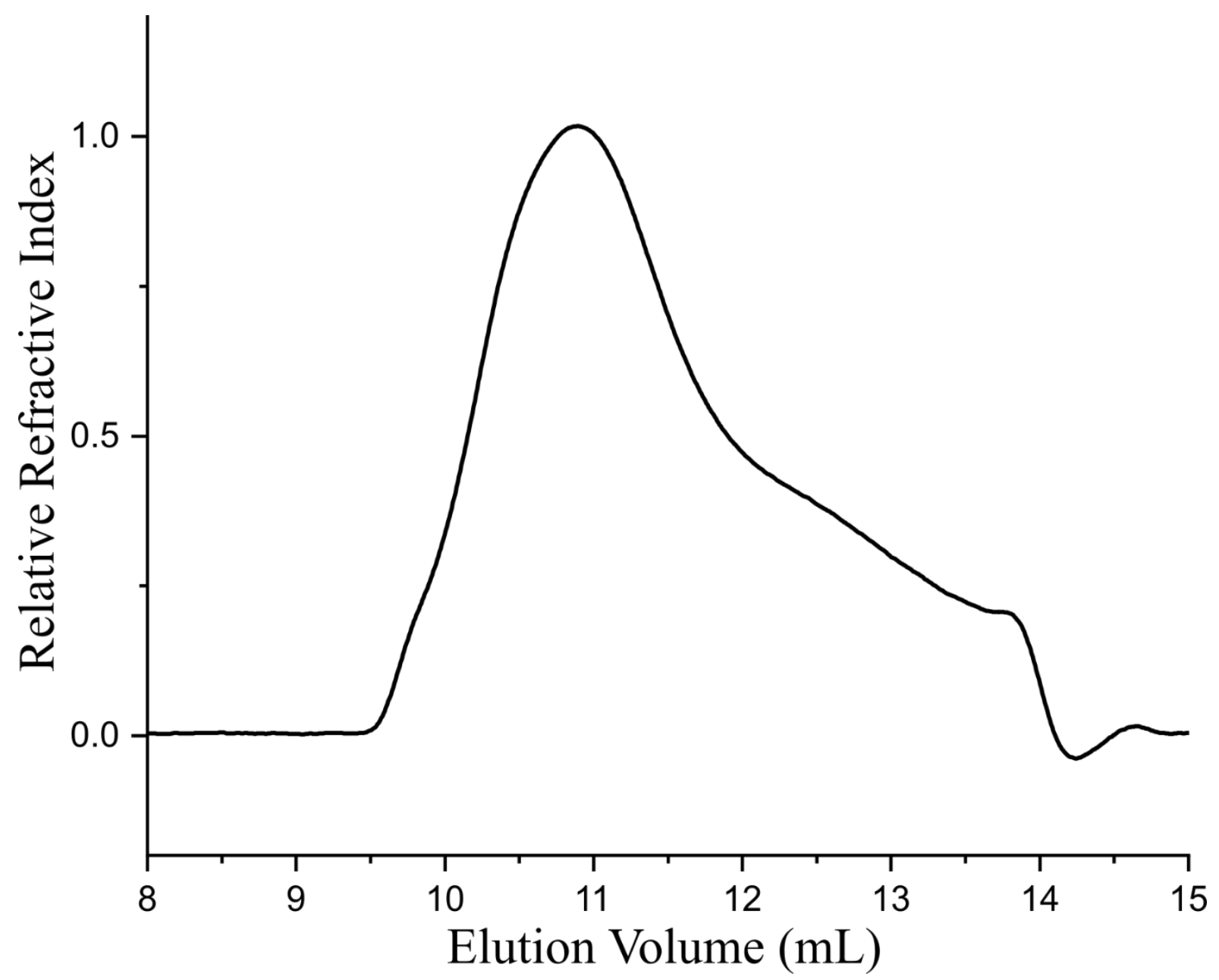

Figure S28. SEC trace of $\mathrm{pDTT}_{200}$ in DMF. 
Supporting Information - Synthesis and Closed-Loop Recycling of Self-Immolative Poly(Dithiothreitol)

Spectrum of pDTT 20 precipitated from $T H F\left({ }^{1} H-N M R\right)$

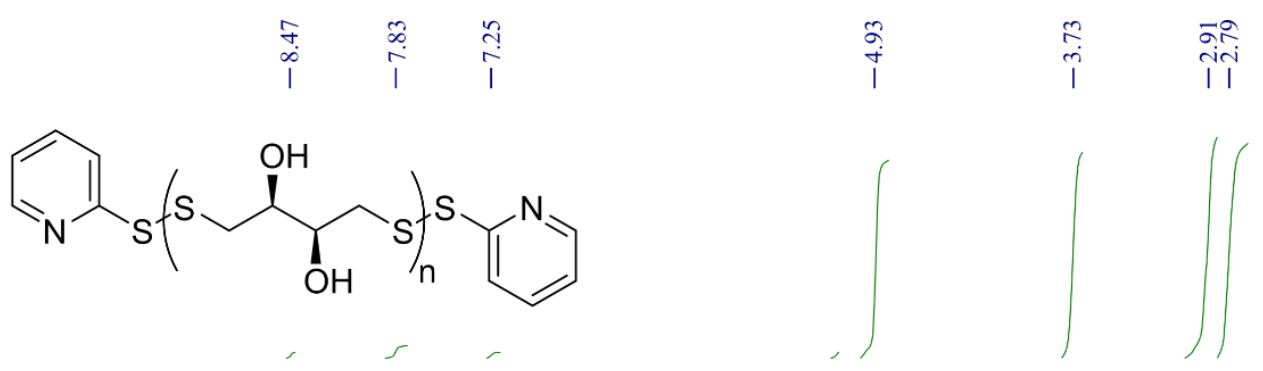

- $\mathrm{CHCl}_{3}$

- THF

DMSO- $d_{6}$

- $\mathrm{H}_{2} \mathrm{O}$

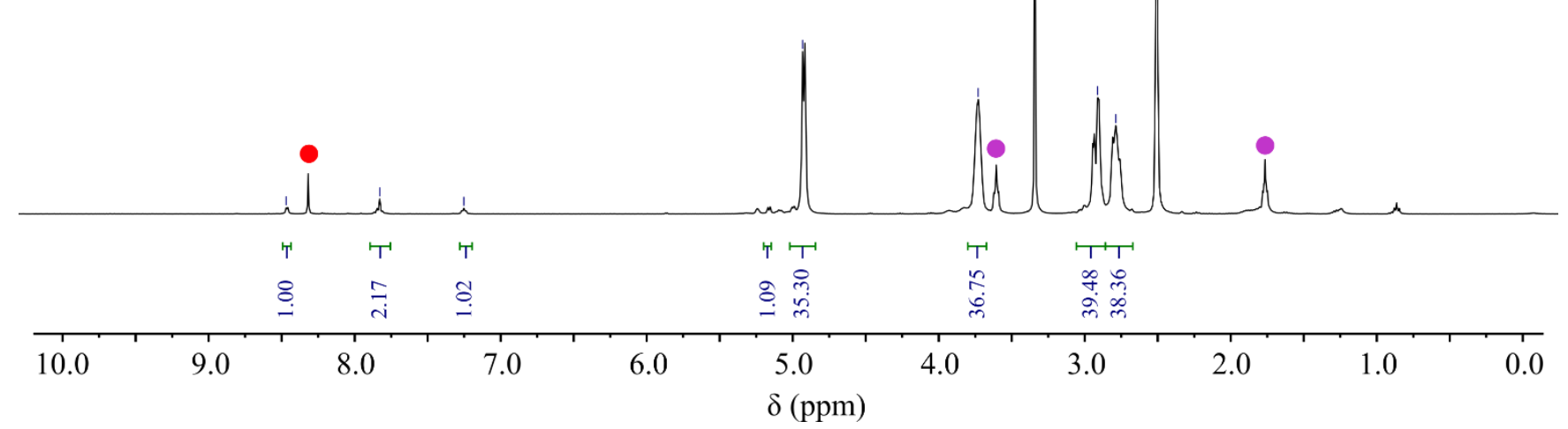

Figure S29. ${ }^{1} \mathrm{H}-\mathrm{NMR}$ spectrum of $\mathrm{pDTT}_{20}$, precipitated from THF to remove DMF residues, in DMSO- $d_{6}$, 
Supporting Information - Synthesis and Closed-Loop Recycling of Self-Immolative Poly(Dithiothreitol)

Degradation of pDTT 20 in solution induced by $60{ }^{\circ} \mathrm{C}$ heat $\left({ }^{1} \mathrm{H}-\mathrm{NMR}\right)$

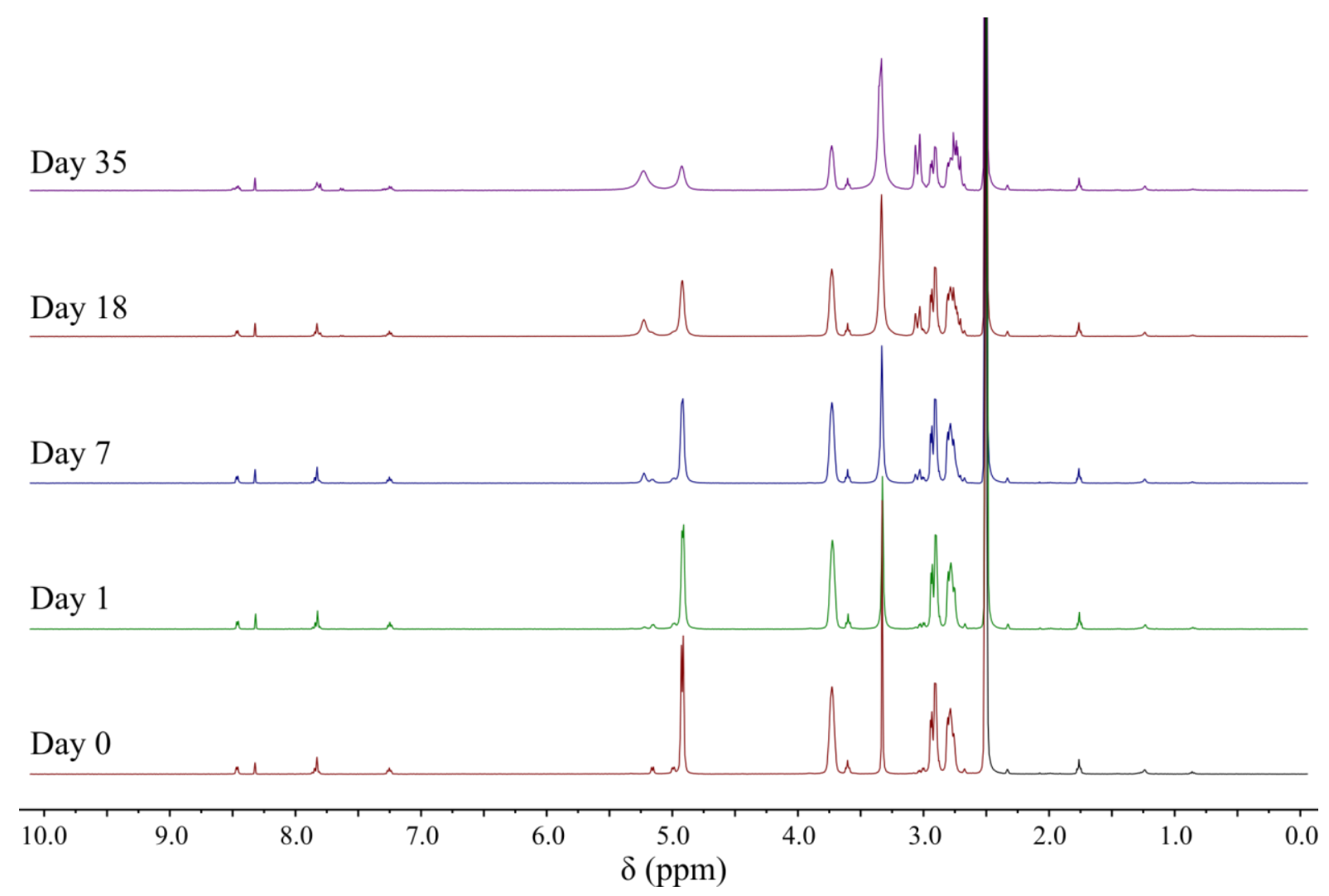

Figure S30. ${ }^{1} \mathrm{H}-\mathrm{NMR}$ spectra of $\mathrm{pDTT}_{20}$ (precipatated from THF and kept at $60{ }^{\circ} \mathrm{C}$ ) recorded at specified time intervals in DMSO- $d_{6}$. Residues of THF released from pDTT 20 are apparent from the beginning $(\delta=3.60$ and 1.76, ppm), in addition to peaks from DMSO- $d_{6}(2.50 \mathrm{ppm}), \mathrm{CHCl}_{3}(8.32 \mathrm{ppm})$ and water $(3.33 \mathrm{ppm})$. 
Supporting Information - Synthesis and Closed-Loop Recycling of Self-Immolative Poly(Dithiothreitol)

Spectra of pDTT $T_{10-200}\left({ }^{1} H-N M R\right)$

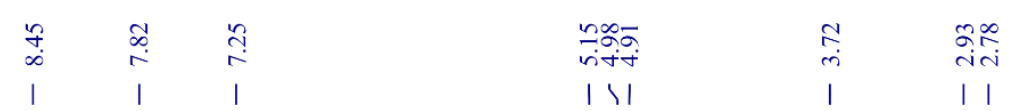

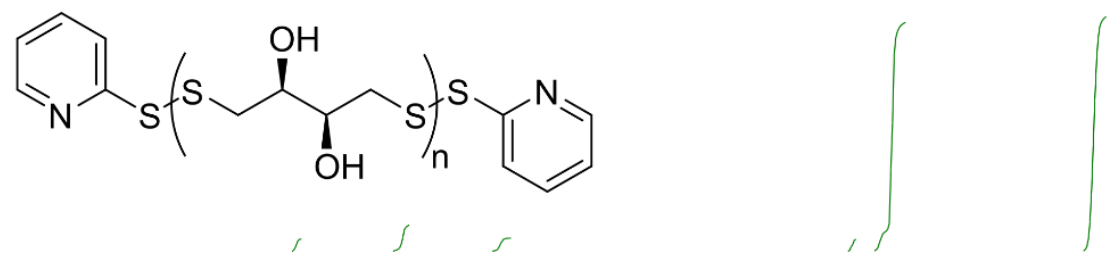

- DMF

- $\mathrm{CHCl}_{3}$

- $\mathrm{DMSO}-d_{6}$

- $\mathrm{H}_{2} \mathrm{O}$

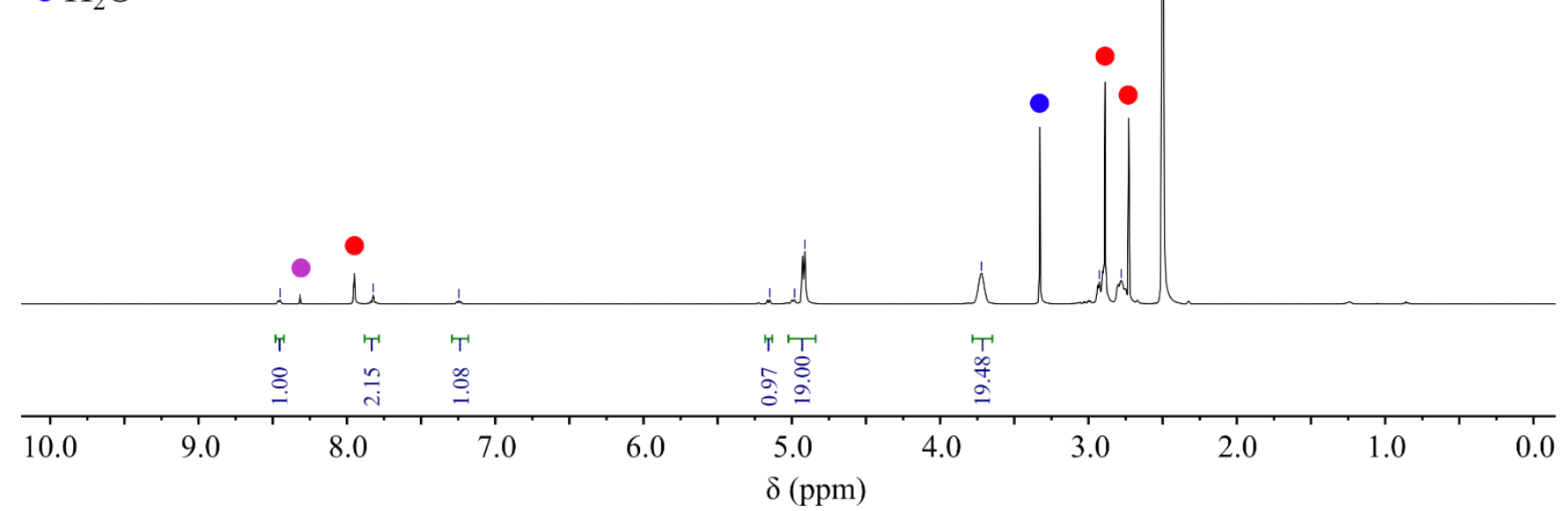

Figure S31. ${ }^{1} \mathrm{H}-\mathrm{NMR}$ spectrum of $\mathrm{pDTT}_{10}$ in DMSO- $d_{6}$. Peaks originating from solvent residues (DMF, $\mathrm{CHCl}_{3}$ and $\mathrm{H}_{2} \mathrm{O}$ ) from the pDTT synthesis are marked. 
Supporting Information - Synthesis and Closed-Loop Recycling of Self-Immolative Poly(Dithiothreitol)

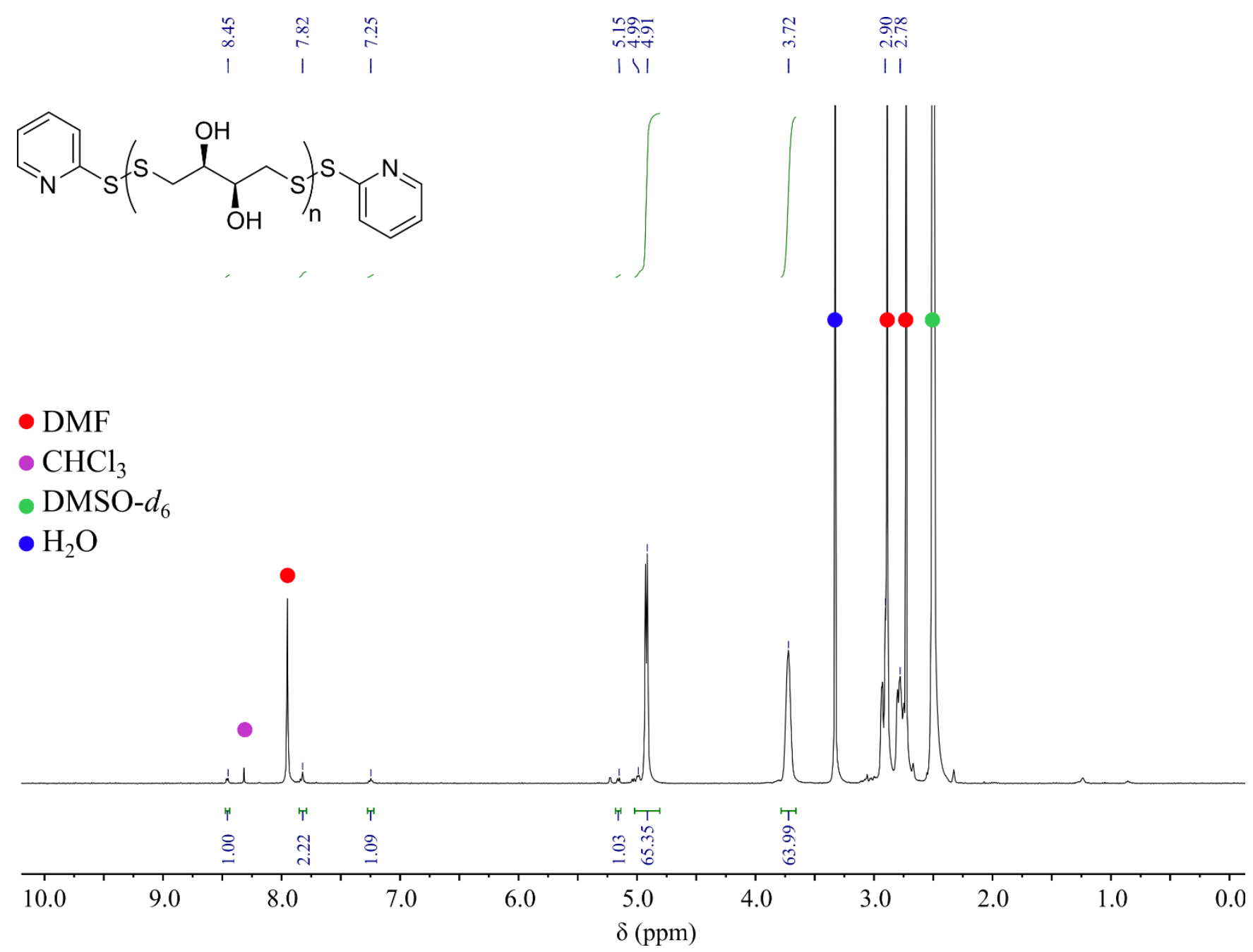

Figure S32. ${ }^{1} \mathrm{H}-\mathrm{NMR}$ spectrum of $\mathrm{pDTT}_{40}$ in DMSO- $d_{6}$. Peaks originating from solvent residues $\left(\mathrm{DMF}, \mathrm{CHCl}_{3}\right.$ and $\left.\mathrm{H}_{2} \mathrm{O}\right)$ from the pDTT synthesis are marked. 
Supporting Information - Synthesis and Closed-Loop Recycling of Self-Immolative Poly(Dithiothreitol)

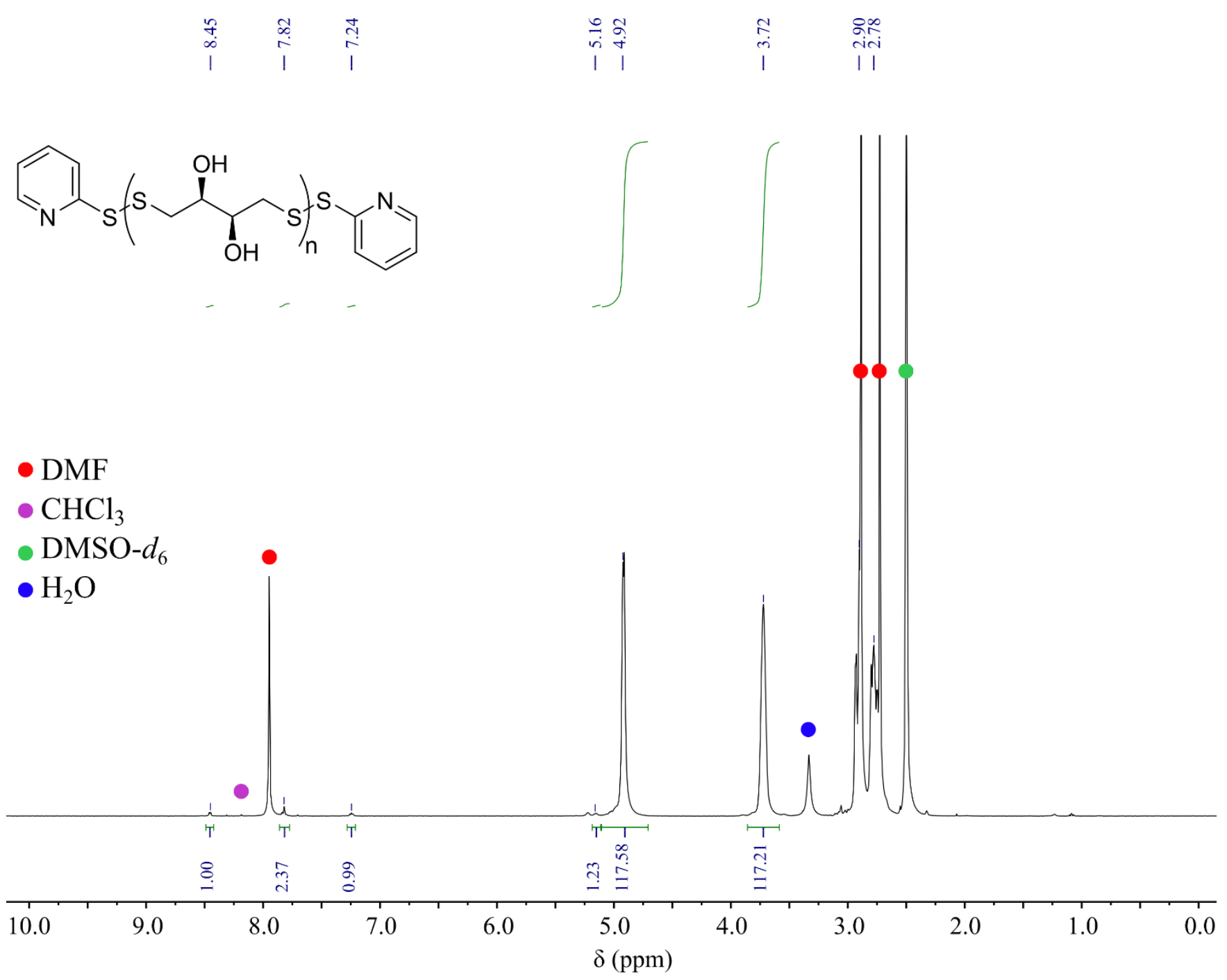

Figure S33. ${ }^{1} \mathrm{H}-\mathrm{NMR}$ spectrum of $\mathrm{pDTT}_{100}$ in DMSO- $d_{6}$. Peaks originating from solvent residues $\left(\mathrm{DMF}, \mathrm{CHCl}_{3}\right.$ and $\left.\mathrm{H}_{2} \mathrm{O}\right)$ from the pDTT synthesis are marked. 
Supporting Information - Synthesis and Closed-Loop Recycling of Self-Immolative Poly(Dithiothreitol)

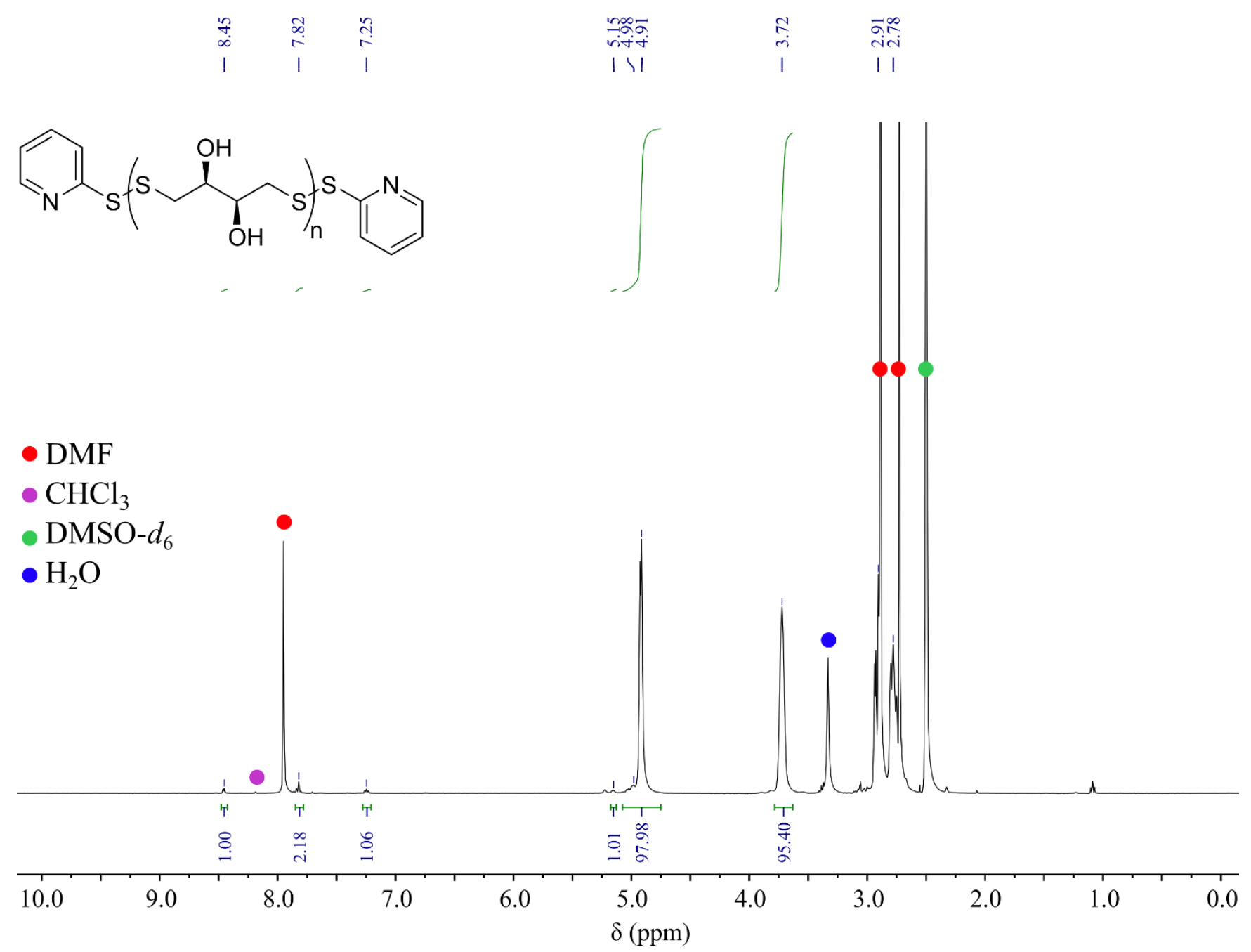

Figure S34. ${ }^{1} \mathrm{H}-\mathrm{NMR}$ spectrum of $\mathrm{pDTT}_{200}$ in DMSO- $d_{6}$. Peaks originating from solvent residues $\left(\mathrm{DMF}, \mathrm{CHCl}_{3}\right.$ and $\left.\mathrm{H}_{2} \mathrm{O}\right)$ from the pDTT synthesis are marked. 
Supporting Information - Synthesis and Closed-Loop Recycling of Self-Immolative Poly(Dithiothreitol)

\section{References}

S1. Canadell, J.; Goossens, H.; Klumperman, B., Self-Healing materials based on disulfide links. Macromolecules 2011, 44, 2536-2541.

S2. Lafont, U.; van Zeijl, H.; van der Zwaag, S., Influence of cross-linkers on the cohesive and adhesive selfhealing ability of polydisulfide-based thermosets. ACS Appl. Mater. 2012, 4, 6280-6288.

S3. $\quad$ Fairbanks, B. D.; Singh, S. P.; Bowman, C. N.; Anseth, K. S., Photodegradable, photoadaptable hydrogels via radical-mediated disulfide fragmentation reaction. Macromolecules 2011, 44, 2444-2450.

S4. Otsuka, H.; Nagano, S.; Kobashi, Y.; Maeda, T.; Takahara, A., A dynamic covalent polymer driven by disulfide metathesis under photoirradiation. Chem. Commun. 2010, 46, 1150-1152.

S5. $\quad$ Dmitrenko, O.; Thorpe, C.; Bach, R. D., Mechanism of SN2 Disulfide Bond Cleavage by Phosphorus Nucleophiles. Implications for Biochemical Disulfide Reducing Agents. J. Org. Chem 2007, 72, $8298-8307$.

\section{Author Contributions}

K.D. conceived the work. S.P., A.S., S.U.P., and K.D. designed the experiments. S.P., A.S., and M.B.D. performed the experiments. A.S., S.U.P., and K.D. wrote the main manuscript. M.H. contributed with equipment and discussions. All authors reviewed the manuscript. 